SPIN-08-38, ITP-UU-08/48, UFIFT-QG-08-06

\title{
Infrared Propagator Corrections for Constant Deceleration
}

\author{
T. M. Janssen*, S. P. Miao**, T. Prokopec ${ }^{\ddagger}$ \\ Institute for Theoretical Physics 83 Spinoza Institute, Utrecht University, \\ Leuvenlaan 4, Postbus 80.195, 3508 TD Utrecht, THE NETHERLANDS \\ R. P. Woodard ${ }^{\dagger \dagger}$ \\ Department of Physics, University of Florida \\ Gainesville, FL 32611, UNITED STATES.
}

\begin{abstract}
We derive the propagator for a massless, minimally coupled scalar on a $D$-dimensional, spatially flat, homogeneous and isotropic background with arbitrary constant deceleration parameter. Our construction uses the operator formalism, by integrating the Fourier mode sum. We give special attention to infrared corrections from the nonzero lower limit associated with working on finite spatial sections. These corrections eliminate infrared divergences that would otherwise be incorrectly treated by dimensional regularization, resulting in offcoincidence divergences for those special values of the deceleration parameter at which the infrared divergence is logarithmic. As an application we compute the expectation value of the scalar stress-energy tensor.
\end{abstract}

PACS numbers: 04.30.-m, 04.62.+v, 98.80.Cq

*T.M.Janssen@uu.nl,

** S.Miao@uu.nl

‡.Prokopec@uu.nl,

†† woodard@phys.ufl.edu 


\section{Introduction}

On the largest scales, the visible universe is well described by a homogeneous, isotropic and spatially flat geometry whose invariant element takes the form,

$$
d s^{2} \equiv g_{\mu \nu} d x^{\mu} d x^{\nu}=-d t^{2}+a^{2}(t) d \vec{x} \cdot d \vec{x} .
$$

Two derivatives of the scale factor $a(t)$ have great importance, the Hubble parameter $H(t)$ and the deceleration parameter $q(t)$,

$$
H(t) \equiv \frac{\dot{a}}{a} \quad, \quad q(t) \equiv-1-\frac{\dot{H}}{H^{2}} \equiv-1+\epsilon(t) .
$$

Although the Hubble parameter may have changed by as much as 57 orders of magnitude from primordial inflation to now, the deceleration parameter is only thought to have varied from nearly -1 , during primordial inflation, to nearly +1 , during the epoch of radiation domination. And $q(t)$ has been approximately constant for vast periods of cosmological evolution. It is therefore interesting to consider physics during an epoch of constant $q(t)$.

A field of great importance for primordial inflation is the massless, minimally coupled scalar,

$$
\mathcal{L}=-\frac{1}{2} \partial_{\mu} \varphi \partial_{\nu} \varphi g^{\mu \nu} \sqrt{-g}=\frac{1}{2} \dot{\varphi}^{2} a^{D-1}-\frac{1}{2}\|\vec{\nabla} \varphi\|^{2} a^{D-3} .
$$

This is true both as an approximation to the inflaton, on account of the very flat potential, and also because dynamical gravitons have the same kinetic operator [1]. Note that we are assuming the spatial manifold is $(D-1)$-dimensional in order to facilitate the use of dimensional regularization.

The scalar propagator obeys the equation,

$$
\sqrt{-g} \square i \Delta\left(x ; x^{\prime}\right) \equiv \partial_{\mu}\left(\sqrt{-g} g^{\mu \nu} \partial_{\nu} i \Delta\left(x ; x^{\prime}\right)\right)=i \delta^{D}\left(x-x^{\prime}\right) .
$$

An elegant solution to this equation has recently been obtained for the case of arbitrary constant $\epsilon \equiv-\dot{H} / H^{2}$, which of course means constant deceleration [2]. This solution has been used to compute the one loop correction to the effective field equations in Einstein + Scalar [3, 4]. Unfortunately, the solution diverges at certain values of $\epsilon$, even off coincidence and with the dimensional regularization in effect. The purpose of this paper is to elucidate the physical basis of this divergence and to provide a very simple correction for it.

It is important to recognize that the propagator equation (4) only fixes $i \Delta\left(x ; x^{\prime}\right)$ up to the addition of a homogeneous solution. There is no guarantee that a particular solution can be interpreted as the expectation value of the time-ordered product of $\varphi(x) \times \varphi\left(x^{\prime}\right)$ in 
the presence of any state. One can see this even from the simple harmonic oscillator of nonrelativistic quantum mechanics [5]. The Heisenberg position operator can be expressed in terms of the initial position and momentum,

$$
q(t)=q_{0} \cos (\omega t)+\frac{p_{0}}{m \omega} \sin (\omega t) .
$$

The associated propagator in the presence of an arbitrary state $|\psi\rangle$ can be expressed in terms of three real numbers,

$$
\begin{aligned}
\left\langle\psi\left|T\left[q(t) q\left(t^{\prime}\right)\right]\right| \psi\right\rangle & =-\frac{i}{2 m \omega} \sin \left(\omega\left|t-t^{\prime}\right|\right) \\
& +\alpha \cos (\omega t) \cos \left(\omega t^{\prime}\right)+\beta \sin \left[\omega\left(t+t^{\prime}\right)\right]+\gamma \sin (\omega t) \sin \left(\omega t^{\prime}\right) .
\end{aligned}
$$

The parameters $\alpha, \beta$ and $\gamma$ are,

$$
\alpha \equiv\left\langle\psi\left|q_{0}^{2}\right| \psi\right\rangle \quad, \quad \beta \equiv \frac{1}{2 m \omega}\left\langle\psi\left|q_{0} p_{0}+p_{0} q_{0}\right| \psi\right\rangle \quad, \quad \gamma \equiv \frac{1}{m^{2} \omega^{2}}\left\langle\psi\left|p_{0}^{2}\right| \psi\right\rangle .
$$

These parameters are not arbitrary. For example, the Uncertainty Principle implies,

$$
\alpha \gamma \geq \frac{1}{4 m^{2} \omega^{2}}
$$

However, the right hand side of (6) solves the propagator equation for any choice of $\alpha, \beta$ and $\gamma$,

$$
-m\left(\frac{d^{2}}{d t^{2}}+\omega^{2}\right) i \Delta\left(t ; t^{\prime}\right)=i \delta\left(t-t^{\prime}\right) .
$$

We seek a true propagator, rather than just a Green's function that solves (41). To ensure this we construct $i \Delta\left(x ; x^{\prime}\right)$ from its operator mode sum in Section 2, assuming that the spatial manifold is $\mathbb{R}^{D-1}$. This gives precisely the result obtained previously [2]. In Section 3 we note that the infinite space mode sum has an infrared divergence for any value of $\epsilon \leq 2(D-1) / D$. In most cases this is a power-law divergence that the automatic subtraction of dimensional regularization simply but erroneously discards. For certain special values of $\epsilon$ the divergence becomes logarithmic, in which case the solution is infinite, even off coincidence and for general $D$. In Section 4 we solve the problem by formulating the mode sum on a finite spatial manifold. This changes the propagator by the addition of a series of homogeneous solutions which cancels the divergences. Section 5 uses the corrected propagator to compute the expectation value of the scalar stress tensor. Our discussion comprises Section 6 . 


\section{Infinite Space Mode Sum}

The purpose of this section is to construct $i \Delta\left(x ; x^{\prime}\right)$ from the canonical operator formalism, assuming the spatial manifold is $\mathbb{R}^{D-1}$. We begin by working out the Hubble parameter and scale factor as functions of co-moving and conformal time. Then we give the free field expansion of $\varphi(x)$. Taking the expectation value of the time-ordered product of $\varphi(x) \times \varphi\left(x^{\prime}\right)$ gives a Fourier integral expression for the propagator which we are able to evaluate by analytically continuing some integral identities. The section closes by noting the special values of $\epsilon$ for which the resulting propagator diverges, away from coincidence and with dimensional regularization in effect.

It is simple to reconstruct the Hubble parameter and the scale factor when $\epsilon$ is constant. If we define $H_{0} \equiv H(0)$ and $a(0) \equiv 1$ then relation (2) implies,

$$
H(t)=\frac{H_{0}}{1+\epsilon H_{0} t} \quad \text { and } \quad a(t)=\left[1+\epsilon H_{0} t\right]^{\frac{1}{\epsilon}} .
$$

The conformal time $\eta$ is defined by $d \eta=d t / a(t)$. We can choose the zero of conformal time so that the following relations apply,

$$
H(\eta)=\frac{H_{0}}{\left[-(1-\epsilon) H_{0} \eta\right]^{\frac{-\epsilon}{1-\epsilon}}} \quad \text { and } \quad a(\eta)=\frac{1}{\left[-(1-\epsilon) H_{0} \eta\right]^{\frac{1}{1-\epsilon}}}
$$

For $0 \leq \epsilon<1$ the universe is accelerating and conformal time approaches zero from below; for $\epsilon>1$ the universe is decelerating and $\eta$ is positive with our convention. A very useful relation is,

$$
(1-\epsilon) H a=-\frac{1}{\eta}
$$

The massless, minimally coupled scalar on $\mathbb{R}^{D-1}$ can be expressed as a Fourier integral of plane waves,

$$
\varphi_{\infty}(\eta, \vec{x})=\int \frac{d^{D-1} k}{(2 \pi)^{D-1}}\left\{u(\eta, k) e^{i \vec{k} \cdot \vec{x}} \alpha(\vec{k})+u^{*}(\eta, k) e^{-i \vec{k} \cdot \vec{x}} \alpha^{\dagger}(\vec{k})\right\}
$$

Since $\varphi_{\infty}$ is a minimally coupled massless scalar, it obeys the Klein Gordon equation $\square \varphi_{\infty}=$ 0 . The mode functions are quite complicated for general, time-dependent $\epsilon(t)[6$. However, for constant $\epsilon$ they obey the simple equation

$$
\left(-\partial_{\eta}^{2}-k^{2}+\frac{\nu^{2}-\frac{1}{4}}{\eta^{2}}\right)\left(a^{\frac{D}{2}-1} u(\eta, k)\right)=0, \quad \nu \equiv \frac{D-1-\epsilon}{2(1-\epsilon)},
$$


where $k=\|\vec{k}\|$. The solution to this equation takes in this case a simple form

$$
u(\eta, k)=\sqrt{\frac{\pi|\eta|}{4}} a^{1-\frac{D}{2}} H_{\nu}^{(1)}(k|\eta|) .
$$

The creation and annihilation operators are canonically normalized,

$$
\left[\alpha(\vec{k}), \alpha^{\dagger}\left(\vec{k}^{\prime}\right)\right]=(2 \pi)^{D-1} \delta^{D-1}\left(\vec{k}-\vec{k}^{\prime}\right) .
$$

The state $|\Omega\rangle$ which is annihilated by $\alpha(\vec{k})$ is known as Bunch-Davies vacuum [7]. It corresponds to the Heisenberg state of minimum excitation in the distant past.

The infinite space propagator is easy to write as a mode sum,

$$
\begin{aligned}
& i \Delta_{\infty}\left(x ; x^{\prime}\right) \equiv\left\langle\Omega\left|\theta\left(t-t^{\prime}\right) \varphi_{\infty}(x) \varphi_{\infty}\left(x^{\prime}\right)+\theta\left(t^{\prime}-t\right) \varphi_{\infty}\left(x^{\prime}\right) \varphi_{\infty}(x)\right| \Omega\right\rangle, \\
& =\frac{\pi}{4} \sqrt{\eta \eta^{\prime}}\left(a a^{\prime}\right)^{1-\frac{D}{2}} \int \frac{d^{D-1} k}{(2 \pi)^{D-1}} e^{i \vec{k} \cdot\left(\vec{x}-\vec{x}^{\prime}\right)} \\
& \quad \times\left\{\theta(\Delta \eta) H_{\nu}^{(1)}(k|\eta|) H_{\nu}^{(1)}\left(k\left|\eta^{\prime}\right|\right)^{*}+\theta(-\Delta \eta) H_{\nu}^{(1)}(k|\eta|)^{*} H_{\nu}^{(1)}\left(k\left|\eta^{\prime}\right|\right)\right\},
\end{aligned}
$$

where $\Delta \eta=\eta-\eta^{\prime}$. Now recall the $D=4$ angular integral,

$$
\int \frac{d^{3} k}{(2 \pi)^{3}} e^{i \vec{k} \cdot \Delta \vec{x}} f(\|\vec{k}\|)=\frac{1}{2 \pi^{2}} \int_{0}^{\infty} d k k^{2} \frac{\sin (k \Delta x)}{k \Delta x} f(k) .
$$

Here and henceforth we define $\Delta x \equiv\left\|\vec{x}-\vec{x}^{\prime}\right\|$. Generalizing to $D$ spacetime dimensions we have $d^{D-1} k=k^{D-2} d k d \Omega_{D-2}, k=\|\vec{k}\|$, where

$$
d \Omega_{D-2}=\sin ^{D-3}\left(\theta_{D-3}\right) d \theta_{D-3} \sin ^{D-4}\left(\theta_{D-4}\right) d \theta_{D-4} \ldots d \phi
$$

where $\theta_{D-3}, \theta_{D-4}, \ldots$, and $\phi$ are the angles on the sphere $\mathbb{S}^{D-2}$. Making use of

$$
\int d \Omega_{D-2}=\frac{2 \pi^{\frac{D-1}{2}}}{\Gamma\left(\frac{D-1}{2}\right)}=\frac{2(4 \pi)^{\frac{D}{2}-1} \Gamma\left(\frac{D}{2}\right)}{\Gamma(D-1)}
$$

and Eq. (8.411.7) in [8] gives,

$$
\int \frac{d^{D-1} k}{(2 \pi)^{D-1}} e^{i \vec{k} \cdot \Delta \vec{x}} f(\|\vec{k}\|)=\frac{1}{2^{D-2} \pi^{\frac{D-1}{2}}} \int_{0}^{\infty} d k k^{D-2} \frac{J_{\frac{D-3}{2}}(k \Delta x)}{\left(\frac{1}{2} k \Delta x\right)^{\frac{D-3}{2}}} f(k) .
$$


Hence the scalar propagator can be reduced to a single integral,

$$
\begin{aligned}
i \Delta_{\infty}\left(x ; x^{\prime}\right) & =\frac{\sqrt{\eta \eta^{\prime}}\left(a a^{\prime}\right)^{1-\frac{D}{2}}}{2^{D} \pi^{\frac{D-3}{2}}} \int_{0}^{\infty} d k k^{D-2} \frac{J_{\frac{D-3}{2}}(k \Delta x)}{\left(\frac{1}{2} k \Delta x\right)^{\frac{D-3}{2}}} \\
& \times\left\{\theta(\Delta \eta) H_{\nu}^{(1)}(k|\eta|) H_{\nu}^{(1)}\left(k\left|\eta^{\prime}\right|\right)^{*}+\theta(-\Delta \eta) H_{\nu}^{(1)}(k|\eta|)^{*} H_{\nu}^{(1)}\left(k\left|\eta^{\prime}\right|\right)\right\} .
\end{aligned}
$$

To reach the final form we make the change of variable,

$$
z \equiv \sqrt{\eta \eta^{\prime}} k \Longrightarrow k \Delta x=\frac{\Delta x}{\sqrt{\eta \eta^{\prime}}} z \quad, \quad k|\eta|=\sqrt{\frac{\eta}{\eta^{\prime}}} z \quad, \quad k\left|\eta^{\prime}\right|=\sqrt{\frac{\eta^{\prime}}{\eta}} z .
$$

Recalling that $1 /(\eta a)=-(1-\epsilon) H$ gives,

$$
\begin{aligned}
i \Delta_{\infty}(x ; & \left.x^{\prime}\right)=\frac{\left(\eta \eta^{\prime} a a^{\prime}\right)^{1-\frac{D}{2}}}{2^{D} \pi^{\frac{D-3}{2}}} \int_{0}^{\infty} d z z^{D-2} \times \frac{J_{\frac{D-3}{2}}\left(\frac{\Delta x}{\sqrt{\eta \eta^{\prime}}} z\right)}{\left(\frac{1}{2} \frac{\Delta x}{\sqrt{\eta \eta^{\prime}}} z\right)^{\frac{D-3}{2}}} \\
& \times\left\{\theta(\Delta \eta) H_{\nu}^{(1)}\left(\sqrt{\frac{\eta}{\eta^{\prime}}} z\right) H_{\nu}^{(1)}\left(\sqrt{\frac{\eta^{\prime}}{\eta}} z\right)^{*}+\theta(-\Delta \eta) \times(\text { conjugate })\right\}, \\
= & {\left[(1-\epsilon)^{2} H H^{\prime}\right]^{\frac{D}{2}-1} \times \frac{\pi^{\frac{3}{2}} 2^{\frac{D-3}{2}}}{\left(\frac{\Delta x}{\sqrt{\eta \eta^{\prime}}}\right)^{\frac{D-3}{2}}} \times \int_{0}^{\infty} d z z^{\frac{D-1}{2}} \times J_{\frac{D-3}{2}}\left(\frac{\Delta x}{\sqrt{\eta \eta^{\prime}}} z\right) } \\
& \times\left\{\theta(\Delta \eta) H_{\nu}^{(1)}\left(\sqrt{\frac{\eta}{\eta^{\prime}}} z\right) H_{\nu}^{(1)}\left(\sqrt{\frac{\eta^{\prime}}{\eta}} z\right)^{*}+\theta(-\Delta \eta) \times(\text { conjugate })\right\} .
\end{aligned}
$$

By analytic continuation of relation (6.578.10) and employing relations (8.702), (8.407.1), (8.476.10) and (9.131.1) in [8] one can show,

$$
\begin{aligned}
\int_{0}^{\infty} d x x^{\mu+1} J_{\mu}(c x) & H_{\nu}^{(1)}(a x) H_{\nu}^{(1)}(b x)^{*}=\frac{\Gamma(\mu+1+\nu) \Gamma(\mu+1-\nu)}{\pi^{\frac{3}{2}} \Gamma\left(\mu+\frac{3}{2}\right)} \\
& \times \frac{\left(\frac{1}{2} c\right)^{\mu}}{(a b)^{\mu+1}}{ }_{2} F_{1}\left(\mu+1+\nu, \mu+1-\nu ; \mu+\frac{3}{2} ; \frac{(a+b)^{2}-c^{2}}{4 a b}\right) .
\end{aligned}
$$

Now make the following assignments for the various parameters in (27),

$$
\mu \longrightarrow \frac{D-3}{2} \quad, \quad a \longrightarrow \sqrt{\frac{\eta}{\eta^{\prime}}} \quad, \quad b \longrightarrow \sqrt{\frac{\eta^{\prime}}{\eta}} \text { and } c \longrightarrow \frac{\Delta x}{\sqrt{\eta \eta^{\prime}}} .
$$


With these assignments we have,

$$
\frac{(a+b)^{2}-c^{2}}{4 a b}=1-\left(\frac{c^{2}-(a-b)^{2}}{4 a b}\right) \longrightarrow 1-\left(\frac{\Delta x^{2}-\Delta \eta^{2}}{4 \eta \eta^{\prime}}\right) .
$$

Hence the mode sum (26) for the propagator can be given the simple spacetime expression,

$$
i \Delta_{\infty}\left(x ; x^{\prime}\right)=\frac{\left[(1-\epsilon)^{2} H H^{\prime}\right]^{\frac{D}{2}-1}}{(4 \pi)^{\frac{D}{2}}} \frac{\Gamma\left(\frac{D-1}{2}+\nu\right) \Gamma\left(\frac{D-1}{2}-\nu\right)}{\Gamma\left(\frac{D}{2}\right)}{ }_{2} F_{1}\left(\frac{D-1}{2}+\nu, \frac{D-1}{2}-\nu ; \frac{D}{2} ; 1-\frac{y}{4}\right) .
$$

Here and henceforth we define the quantity $y=y\left(x ; x^{\prime}\right)$ as,

$$
y\left(x ; x^{\prime}\right) \equiv \frac{\left\|\vec{x}-\vec{x}^{\prime}\right\|^{2}-\left(\left|\eta-\eta^{\prime}\right|-i \varepsilon\right)^{2}}{\eta \eta^{\prime}} .
$$

Note the distinction between the infinitesimal quantity $\varepsilon$, used to define the pole prescription, and the parameter $\epsilon \equiv-\dot{H} / H^{2}$. The propagator (30) is the generalization of the ChernikovTagirov propagator for de Sitter space to space-times with constant, but arbitrary $\epsilon$ [9]. The constant $\epsilon$ propagator was already found for $D=4$ in [10].

Expression (30) is precisely the result that was obtained previously by solving the propagator equation (41) with the ansatz of $\left(H H^{\prime}\right)^{\frac{D}{2}-1}$ times a function of $y\left(x ; x^{\prime}\right)$ [3, 4]. If we employ the transformation formulae for hypergeometric functions and then their series expansion we can write this as,

$$
\begin{aligned}
i \Delta_{\infty}\left(x ; x^{\prime}\right)= & \frac{\left[(1-\epsilon)^{2} H H^{\prime}\right]^{\frac{D}{2}-1}}{(4 \pi)^{\frac{D}{2}}}\left\{\Gamma\left(\frac{D}{2}-1\right)\left(\frac{4}{y}\right)^{\frac{D}{2}-1}{ }_{2} F_{1}\left(\frac{1}{2}+\nu, \frac{1}{2}-\nu ; 2-\frac{D}{2} ; \frac{y}{4}\right)\right. \\
& \left.+\frac{\Gamma\left(\frac{D-1}{2}+\nu\right) \Gamma\left(\frac{D-1}{2}-\nu\right) \Gamma\left(1-\frac{D}{2}\right)}{\Gamma\left(\frac{1}{2}+\nu\right) \Gamma\left(\frac{1}{2}-\nu\right)}{ }_{2} F_{1}\left(\frac{D-1}{2}+\nu, \frac{D-1}{2}-\nu ; \frac{D}{2} ; \frac{y}{4}\right)\right\}, \\
= & \frac{\left[(1-\epsilon)^{2} H H^{\prime}\right]^{\frac{D}{2}-1}}{(4 \pi)^{\frac{D}{2}}} \Gamma\left(\frac{D}{2}-1\right)\left\{\left(\frac{4}{y}\right)^{\frac{D}{2}-1}+\frac{\Gamma\left(2-\frac{D}{2}\right)}{\Gamma\left(\frac{1}{2}+\nu\right) \Gamma\left(\frac{1}{2}-\nu\right)}\right. \\
& \left.\times \sum_{n=0}^{\infty}\left[\frac{\Gamma\left(\frac{3}{2}+\nu+n\right) \Gamma\left(\frac{3}{2}-\nu+n\right)}{\Gamma\left(3-\frac{D}{2}+n\right)(n+1) !}\left(\frac{y}{4}\right)^{n-\frac{D}{2}+2}-\frac{\Gamma\left(\frac{D-1}{2}+\nu+n\right) \Gamma\left(\frac{D-1}{2}-\nu+n\right)}{\Gamma\left(\frac{D}{2}+n\right) n !}\left(\frac{y}{4}\right)^{n}\right]\right\} .
\end{aligned}
$$


The problem with (33) is that the gamma functions on the last line diverge for certain values of $\epsilon$, irrespective of whether or not $x^{\prime \mu}=x^{\mu}$ and with the dimensional regularization still in effect. For the inflationary case $0 \leq \epsilon<1$ divergences occur at,

$$
\epsilon=\frac{2 N}{D-2+2 N} \Longrightarrow \Gamma\left(\frac{D-1}{2}-\nu+n\right)=\Gamma(-N+n) \quad \text { for } \quad N=0,1,2, \ldots
$$

For the decelerating case of $1<\epsilon$ divergences are found at,

$$
\epsilon=2 \frac{D-1+N}{D+2 N} \Longrightarrow \Gamma\left(\frac{D-1}{2}+\nu+n\right)=\Gamma(-N+n) \quad \text { for } \quad N=0,1,2, \ldots
$$

\section{Origin of the Problem}

The purpose of this section is to explain why the infinite space propagator (33) diverges for the special values of $\epsilon$ given in (34).35). We begin by discussing infrared divergences of the mode sum (26). The discrete values of $\epsilon$ result from how these infrared divergences are handled by dimensional regularization. Of course an infrared divergence should not be subtracted like an ultraviolet divergence! The correct procedure is to instead identify and remove whatever unphysical feature led to the infrared divergence. We close by doing this for the scalar propagator.

It has long been known that the infinite space mode sum (26) has infrared divergences for broad ranges of constant $\epsilon$ [11]. They follow from the small argument expansions of the Bessel function,

$$
J_{\nu}(z)=\sum_{n=0}^{\infty} \frac{(-1)^{n}\left(\frac{1}{2} z\right)^{\nu+2 n}}{n ! \Gamma(\nu+n+1)}
$$

and from its relation to the Hankel function,

$$
H_{\nu}^{(1)}(z) \equiv J_{\nu}(z)+i N_{\nu}(z)=\frac{i}{\sin (\nu \pi)}\left\{e^{-i \nu \pi} J_{\nu}(z)-J_{-\nu}(z)\right\}
$$

The leading small $z$ behavior for the terms on the first line of (26) is universal,

$$
z^{\frac{D-1}{2}} \times J_{\frac{D-3}{2}}\left(\frac{\Delta x}{\sqrt{\eta \eta^{\prime}}} z\right) \rightarrow \frac{z^{D-2}}{\Gamma\left(\frac{D-1}{2}\right)} \times\left(\frac{\Delta x}{2 \sqrt{\eta \eta^{\prime}}}\right)^{\frac{D-3}{2}}
$$

What we get for the two Hankel functions on the second line depends upon whether the universe is accelerating or decelerating. For the inflationary case of $0 \leq \epsilon<1$ the index $\nu$ is 
positive and it is the $J_{-\nu}$ terms in the Hankel functions which make the leading contributions for small $z$,

$$
H_{\nu}^{(1)}\left(\sqrt{\frac{\eta}{\eta^{\prime}}} z\right) H_{\nu}^{(1)}\left(\sqrt{\frac{\eta^{\prime}}{\eta}} z\right)^{*} \longrightarrow \frac{\left(\frac{1}{2} z\right)^{-2 \nu}}{\sin ^{2}(\nu \pi) \Gamma^{2}(-\nu+1)}=\frac{2^{2 \nu} \Gamma^{2}(\nu)}{\pi^{2}} \times z^{-2 \nu} .
$$

For the decelerating case of $1<\epsilon \leq(D-1)$ the index $\nu$ is negative and it is the $J_{+\nu}$ terms in the Hankel functions which make the leading contributions for small $z$,

$$
H_{\nu}^{(1)}\left(\sqrt{\frac{\eta}{\eta^{\prime}}} z\right) H_{\nu}^{(1)}\left(\sqrt{\frac{\eta^{\prime}}{\eta}} z\right)^{*} \longrightarrow \frac{\left(\frac{1}{2} z\right)^{2 \nu}}{\sin ^{2}(\nu \pi) \Gamma^{2}(\nu+1)}=\frac{2^{-2 \nu} \Gamma^{2}(-\nu)}{\pi^{2}} \times z^{2 \nu}
$$

From the preceding discussion we see that the asymptotic small $z$ form of the integrand in (26) is a constant times,

$$
z^{D-2-2|\nu|}
$$

For this to produce an infrared divergence requires the exponent to be -1 or less. For inflationary case of $0 \leq \epsilon<1$ we have $\nu>0$ and the condition for a divergence is always met,

$$
0 \leq \epsilon<1 \quad \Longrightarrow \quad-1 \geq D-2-\frac{D-1-\epsilon}{1-\epsilon}=-1-\frac{\epsilon(D-2)}{1-\epsilon} .
$$

For the decelerating case of $1<\epsilon<(D-1)$ we have $\nu<0$ and the condition is met as long as $\epsilon \leq 2(D-1) / D$,

$$
1<\epsilon \leq \frac{2(D-1)}{D} \Longrightarrow-1 \geq D-2+\frac{D-1-\epsilon}{1-\epsilon}=-1-\frac{2(D-1)-D \epsilon}{\epsilon-1} .
$$

Although the mode sum (26) has infrared divergences for all $0 \leq \epsilon \leq 2(D-1) / D$, the final result (33) only diverges for the discrete values of $\epsilon$ given by expressions (34 35). This is because dimensional regularization [12, 13] automatically subtracts power law divergences and only registers logarithmic divergences. For most values of $\epsilon$ the infrared divergence is a power law, and dimensional regularization — quite incorrectly — sets it to zero. It is only for the discrete values (34) that a logarithmic divergence occurs and causes expression (33) to become ill-defined. To see this, note that the logarithmic divergence could derive from any of the order $z^{2 N}$ series corrections to the leading small $z$ term. For the inflationary range of $0 \leq \epsilon<1$ the condition for a logarithmic infrared divergence corresponds precisely to (34),

$$
\begin{aligned}
0 \leq \epsilon<1 & \Longrightarrow(D-2)+2 N-\frac{D-1-\epsilon}{1-\epsilon}=-1 \\
& \Longrightarrow \epsilon=\frac{2 N}{D-2+2 N} \quad \text { for } \quad N=0,1,2, \ldots
\end{aligned}
$$


In $D=4$ dimensions the problem values are $\epsilon=0, \frac{1}{2}, \frac{2}{3}, \frac{3}{4}, \ldots$ For the decelerating case of $1<\epsilon \leq 2(D-1) / D$ the condition for a logarithmic infrared divergence agrees with (35),

$$
\begin{aligned}
1<\epsilon \leq \frac{2(D-1)}{D} & \Longrightarrow(D-2)+2 N+\frac{D-1-\epsilon}{1-\epsilon}=-1, \\
& \Longrightarrow \epsilon=2 \frac{D-1+N}{D+2 N} \quad \text { for } \quad N=0,1,2, \ldots
\end{aligned}
$$

For $D=4$ this corresponds to $\epsilon=\frac{3}{2}, \frac{4}{3}, \frac{5}{4}, \ldots$

It is important to understand that the infinite space propagator has physical problems for every value of $\epsilon$ in the infrared divergent range $0 \leq \epsilon \leq 2(D-1) / D$, whether or not $\epsilon$ happens to take one of the critical values (34) necessary for a logarithmic divergence. This is because ultraviolet and infrared divergences mean different things. Ultraviolet divergences indicate that loop corrections have made an infinite change between observed parameters and the corresponding parameters of the Lagrangian. They can be canceled by expressing the parameters of the Lagrangian in terms of observed quantities plus counterterms which subtract off the divergences [14]. The automatic subtraction of dimensional regularization is not an error for ultraviolet divergences; it merely saves one the trouble of defining and subtracting the appropriate counterterm to cancel a power law divergence.

Infrared divergences do not mean anything about parameters in the Lagrangian. Instead, they signify that there is something wildly unphysical about the computation being done. One does not deal with an infrared divergence by subtracting a counterterm; the correct procedure is rather to compute physically well-defined quantities. The classic example is the Bloch-Nordsieck switch from infrared divergent, exclusive processes to infrared finite, inclusive processes in quantum electrodynamics [14]. It will be seen that employing the automatic subtraction of dimensional regularization to remove a power law infrared divergence corresponds to adding an illegal counterterm to make an unphysical question return a finite answer, instead of reformulating the question in more physical terms.

The unphysical thing about the infinite space propagator is that a local observer cannot prepare the initially super-horizon modes of the state in coherent Bunch-Davies vacuum. Two plausible fixes have been proposed:

- One could work on infinite space as in (13) but assume that the super-horizon modes are less singular than Bunch-Davies vacuum [15]. Because only the super-horizon modes change there would be no effect on the Hadamard behavior of the propagator. If one continues to regard the state as obeying $\alpha(\vec{k})|\Omega\rangle=0$, this fix corresponds to changing the super-horizon mode functions $u(t, k)$ from the Bunch-Davies choice (15). Of course their time dependence is determined by the scalar field equation but their initial values and those of their first time derivatives can be freely specified. For example, if these 
initial values were chosen to be those of the Bunch-Davies mode functions for $\epsilon=D / 2$ (regardless of the actual value of $\epsilon$ ) then there would be no infrared divergence, either initially or at any later time[16].

- One could also work on a compact spatial manifold such as a torus $T^{D-1}$ for which there are no initially super-horizon modes [17]. In this case the free field expansion becomes a sum rather than an integral but it is generally valid to make the integral approximation to this sum, with a nonzero lower limit. When this was done for the graviton propagator on de Sitter background $(\epsilon=0)$ there is no disturbance to powerful consistency checks such as the one loop Ward identity [18] and the nature of allowed counterterms [19, 20]. The renormalization of scalar field theories is not even affected at two loop order [21, 22, 23].

\section{Finite Space Mode Sum}

The purpose of this section is to implement the second of the two fixes described above: the one based upon a finite-sized spatial manifold [17]. We show how this changes the mode sum for the propagator. We also derive the corrections it makes to the integrated, positionspace form. Explicit demonstrations are given that the correction terms cure the $N=0$ and $N=1$ divergences in expressions (34 35). And certain special cases are checked against known results [21, 24.

We work on $T^{D-1}$, which supports the spatially flat FRW geometry (1). If the coordinate radius in each direction is $2 \pi / k_{0}$ then the integral approximation for the free field expansion of the operator is the same as (13) except that the integral is cut off at $\|\vec{k}\|=k_{0}$,

$$
\varphi(t, \vec{x})=\int \frac{d^{D-1} k}{(2 \pi)^{D-1}} \theta\left(k-k_{0}\right)\left\{u(t, k) e^{i \vec{k} \cdot \vec{x}} \alpha(\vec{k})+u^{*}(t, k) e^{-i \vec{k} \cdot \vec{x}} \alpha^{\dagger}(\vec{k})\right\} .
$$

Of course the same cutoff works its way into the mode sum for the propagator (26),

$$
\begin{aligned}
& i \Delta\left(x ; x^{\prime}\right)= \\
& \frac{\left[(1-\epsilon)^{2} H H^{\prime}\right]^{\frac{D}{2}-1}}{(4 \pi)^{\frac{D}{2}}} \frac{\pi^{\frac{3}{2}} 2^{\frac{D-3}{2}}}{\left(\frac{\Delta x}{\sqrt{\eta \eta^{\prime}}}\right)^{\frac{D-3}{2}}} \int_{z_{0}}^{\infty} d z z^{\frac{D-1}{2}} J_{\frac{D-3}{2}}\left(\frac{\Delta x}{\sqrt{\eta \eta^{\prime}}} z\right) \\
& \quad \times\left\{\theta(\Delta \eta) H_{\nu}^{(1)}\left(\sqrt{\frac{\eta}{\eta^{\prime}}} z\right) H_{\nu}^{(1)}\left(\sqrt{\frac{\eta^{\prime}}{\eta}} z\right)^{*}+\theta(-\Delta \eta) \times(\text { conjugate })\right\} .
\end{aligned}
$$


Here and subsequently $z_{0} \equiv k_{0} \sqrt{\eta \eta^{\prime}}$.

We can obviously break the integral over $z$ up into two parts,

$$
\int_{z_{0}}^{\infty} d z=\int_{0}^{\infty} d z-\int_{0}^{z_{0}} d z .
$$

This means that the result for (49) is what we already have (33) from the work of [2, 3, 4], minus the finite range integral. It would be simple enough to expand the integrand of this second contribution and then integrate termwise, but we really only need the most infrared singular parts. For the inflationary case of $0 \leq \epsilon<1$ the index $\nu$ is positive and the most infrared singular parts of the integrand derive from the $J_{-\nu}$ contributions to the Hankel functions. For the decelerating case of $1<\epsilon \leq 2(D-1) / D$ the index $\nu$ is negative and it is the $J_{+\nu}$ parts of the Hankel functions that are the most infrared singular. We shall work out the series of leading corrections in each case.

Let us begin with the most infrared singular correction for the inflationary case of $0 \leq$ $\epsilon<1$. From the small $z$ forms (38) and (39) we see that the desired correction is,

$$
\begin{aligned}
\delta i \Delta_{0}\left(x ; x^{\prime}\right) & =-\frac{\left[(1-\epsilon)^{2} H H^{\prime}\right]^{\frac{D}{2}-1}}{(4 \pi)^{\frac{D}{2}}} \frac{2^{2 \nu} \Gamma^{2}(\nu)}{\pi^{\frac{1}{2}} \Gamma\left(\frac{D-1}{2}\right)} \int_{0}^{z_{0}} d z z^{D-2-2 \nu} \\
& =\frac{\left[(1-\epsilon)^{2} H H^{\prime}\right]^{\frac{D}{2}-1}}{(4 \pi)^{\frac{D}{2}}} \frac{\Gamma(2 \nu) \Gamma(\nu)}{\Gamma\left(\frac{1}{2}+\nu\right) \Gamma\left(\frac{D-1}{2}\right)} \frac{2(1-\epsilon)}{\epsilon(D-2)}\left(\frac{1}{k_{0}^{2} \eta \eta^{\prime}}\right)^{\frac{\epsilon(D-2)}{2(1-\epsilon)}} .
\end{aligned}
$$

To reach the last form (51) we have used the doubling formula for the Gamma function,

$$
\Gamma(2 x)=\frac{2^{2 x-1}}{\pi^{\frac{1}{2}}} \Gamma(x) \Gamma\left(x+\frac{1}{2}\right) .
$$

Of course the infrared divergence at $z=0$ in $\delta i \Delta_{0}$ was dimensionally regulated, the same way as in the infinite space result $i \Delta_{\infty}$. This is wrong for $\delta i \Delta_{0}$, just as it was for $i \Delta_{\infty}$, but expression (50) implies that the two errors must cancel and we will shortly see this explicitly.

We shall use the notation $\delta i \Delta_{N}$ to indicate the $N$-th order correction in the case of an inflationary universe $(\epsilon<1)$. For the correction in the decelerating case $(1<\epsilon \leq 2(D-1) / D)$ we use the notation $\delta i \Delta^{N}$. Before deriving the higher corrections, let us see that the addition of (51) eliminates the $N=0$ divergence (34). Both (51) and the $n=0$ term from the last line of (33) have a common factor that we may as well omit,

$$
\frac{\left[(1-\epsilon)^{2} H H^{\prime}\right]^{\frac{D}{2}-1}}{(4 \pi)^{\frac{D}{2}}} \times \frac{1}{\Gamma\left(\frac{1}{2}+\nu\right)} .
$$


The remaining contributions are,

$$
\begin{aligned}
\frac{2(1-\epsilon) \Gamma(2 \nu) \Gamma(\nu)}{\epsilon(D-2) \Gamma\left(\frac{D-1}{2}\right)}\left(\frac{1}{k_{0}^{2} \eta \eta^{\prime}}\right)^{\frac{\epsilon(D-2)}{2(1-\epsilon)}}-\frac{\Gamma\left(\frac{D}{2}-1\right) \Gamma\left(2-\frac{D}{2}\right)}{\Gamma\left(\frac{1}{2}-\nu\right)} \frac{\Gamma\left(\frac{D-1}{2}+\nu\right) \Gamma\left(\frac{D-1}{2}-\nu\right)}{\Gamma\left(\frac{D}{2}\right)} \\
=\frac{2(1-\epsilon) \Gamma(2 \nu) \Gamma(\nu)}{\epsilon(D-2) \Gamma\left(\frac{D-1}{2}\right)}\left\{\left(\frac{1}{k_{0}^{2} \eta \eta^{\prime}}\right)^{\frac{\epsilon(D-2)}{2(1-\epsilon)}}\right. \\
\left.+\frac{\Gamma\left(\frac{D-1}{2}\right)}{\Gamma(\nu)} \frac{\Gamma\left(1-\frac{D}{2}\right)}{\Gamma\left(\frac{1}{2}-\nu\right)} \frac{\Gamma\left(\frac{D-1}{2}+\nu\right)}{\Gamma(2 \nu)} \frac{\Gamma\left(\frac{D-1}{2}-\nu\right)}{\frac{2(1-\epsilon)}{\epsilon(D-2)}}\right\} .
\end{aligned}
$$

It is convenient to expand this expression in terms of the small parameter $\alpha \equiv \epsilon(D-2) /[2(1-\epsilon)]$ such that in the limit when $\epsilon$ vanishes expression (54) reduces to

$$
\begin{aligned}
& \lim _{\epsilon \rightarrow 0} \frac{2(1-\epsilon) \Gamma(2 \nu) \Gamma(\nu)}{\epsilon(D-2) \Gamma\left(\frac{D-1}{2}\right)}\left\{\left(\frac{1}{k_{0}^{2} \eta \eta^{\prime}}\right)^{\frac{\epsilon(D-2)}{2(1-\epsilon)}}+\frac{\Gamma\left(\frac{D-1}{2}\right)}{\Gamma(\nu)} \frac{\Gamma\left(1-\frac{D}{2}\right)}{\Gamma\left(\frac{1}{2}-\nu\right)} \frac{\Gamma\left(\frac{D-1}{2}+\nu\right)}{\Gamma(2 \nu)} \frac{\Gamma\left(\frac{D-1}{2}-\nu\right)}{\frac{2(1-\epsilon)}{\epsilon(D-2)}}\right\} \\
& =\Gamma(D-1)\left\{\ln \left(a a^{\prime}\right)-\pi \cot \left(\frac{\pi D}{2}\right)+2 \ln \left(\frac{H_{0}}{k_{0}}\right)+\psi\left(\frac{D-1}{2}\right)-\psi\left(\frac{D}{2}\right)+\psi(D-1)-\gamma\right\},
\end{aligned}
$$

where $\psi(z)=(d / d z) \ln (\Gamma(z))$ indicates the digamma function, we used $a=-1 / H_{0} \eta$ (valid for $\epsilon=0), \psi(1)=-\gamma$ and the reflection formula for the digamma function,

$$
\psi(1-x)=\psi(x)+\pi \cot (\pi x)
$$

Multiplying (55) by the common factor (53), and then adding the rest of (133) — which is not singular for $\epsilon=0$ - gives the following result,

$$
\begin{gathered}
\lim _{\epsilon \rightarrow 0} i \Delta\left(x ; x^{\prime}\right)=\frac{H_{0}^{D-2}}{(4 \pi)^{\frac{D}{2}}}\left\{\frac{\Gamma\left(\frac{D}{2}\right)}{\frac{D}{2}-1}\left(\frac{4}{y}\right)^{\frac{D}{2}-1}+\frac{\Gamma\left(\frac{D}{2}+1\right)}{\frac{D}{2}-2}\left(\frac{4}{y}\right)^{\frac{D}{2}-2}+\frac{\Gamma(D-1)}{\Gamma\left(\frac{D}{2}\right)}\left[\ln \left(a a^{\prime}\right)\right.\right. \\
\left.-\pi \cot \left(\frac{\pi D}{2}\right)+2 \ln \left(\frac{H_{0}}{k_{0}}\right)+\psi\left(\frac{D-1}{2}\right)-\psi\left(\frac{D}{2}\right)+\psi(D-1)-\gamma\right] \\
\left.+\sum_{n=1}^{\infty}\left[\frac{\Gamma(D-1+n)}{n \Gamma\left(\frac{D}{2}+n\right)}\left(\frac{y}{4}\right)^{n}-\frac{\Gamma\left(\frac{D}{2}+1+n\right)}{\left(2-\frac{D}{2}+n\right)(n+1) !}\left(\frac{y}{4}\right)^{n-\frac{D}{2}+2}\right]+O\left(k_{0}^{2}\right)\right\} .
\end{gathered}
$$

Except for the order $k_{0}^{2}$ corrections, and for some constant, finite factors on the second line, expression (57) agrees precisely with the result first obtained in [21] and used subsequently in many one and two loop computations [19, 20, 22, 23, 25] on de Sitter background. 
It is straightforward to work out the next contributions from the lower limit. We merely add up the three first order corrections from the Bessel and Hankel functions for the case of $\nu$ positive,

$$
\begin{aligned}
J_{\frac{D-3}{2}}\left(\frac{\Delta x}{\sqrt{\eta \eta^{\prime}}} z\right) & \left.=\left(\frac{\Delta x}{2 \sqrt{\eta \eta^{\prime}}}\right)^{\frac{D-3}{2}} \times \frac{z^{\frac{D-3}{2}}}{\Gamma\left(\frac{D-1}{2}\right)}\left\{1-\frac{\frac{\Delta x^{2}}{\left(\frac{D \eta^{\prime}}{2} z^{2}\right.}}{2}\right)+O\left(z^{4}\right)\right\}, \\
H_{\nu}^{(1)}\left(\sqrt{\frac{\eta}{\eta^{\prime}}} z\right) & =\frac{-i\left(\frac{1}{2} z\right)^{-\nu}\left(\eta / \eta^{\prime}\right)^{-\nu / 2}}{\sin (\nu \pi) \Gamma(1-\nu)}\left\{1-\frac{\frac{\eta}{\eta^{\prime}}}{1-\nu}+O\left(z^{4}\right)\right\}, \\
H_{\nu}^{(1)}\left(\sqrt{\frac{\eta^{\prime}}{\eta}} z\right)^{*} & =\frac{i\left(\frac{1}{2} z\right)^{-\nu}\left(\eta^{\prime} / \eta\right)^{-\nu / 2}}{\sin (\nu \pi) \Gamma(1-\nu)}\left\{1-\frac{\frac{\eta^{\prime}}{\eta} \frac{z^{2}}{4}}{1-\nu}+O\left(z^{4}\right)\right\} .
\end{aligned}
$$

The resulting lower limit term is,

$$
\begin{gathered}
\delta i \Delta_{1}=-\frac{\left[(1-\epsilon)^{2} H H^{\prime}\right]^{\frac{D}{2}-1}}{(4 \pi)^{\frac{D}{2}}} \frac{2 \Gamma(2 \nu) \Gamma(\nu)}{\Gamma\left(\frac{1}{2}+\nu\right) \Gamma\left(\frac{D-1}{2}\right)}\left[\frac{\left(\eta^{2}+\eta^{\prime 2}\right)}{4(\nu-1) \eta \eta^{\prime}}-\frac{\Delta x^{2}}{2(D-1) \eta \eta^{\prime}}\right] \int_{0}^{z_{0}} d z z^{D-2 \nu} \\
=\frac{\left[(1-\epsilon)^{2} H H^{\prime}\right]^{\frac{D}{2}-1}}{(4 \pi)^{\frac{D}{2}}} \frac{2 \Gamma(2 \nu) \Gamma(\nu)}{\Gamma\left(\frac{1}{2}+\nu\right) \Gamma\left(\frac{D-1}{2}\right)} \\
\times\left[\frac{\left(\eta^{2}+\eta^{\prime 2}\right)}{4(\nu-1) \eta \eta^{\prime}}-\frac{\Delta x^{2}}{2(D-1) \eta \eta^{\prime}}\right] \frac{-z_{0}^{D+1-2 \nu}}{D+1-2 \nu}
\end{gathered}
$$

This should cancel the $N=1$ diverge of (34) at $\epsilon=2 / D$, which affects the $n=0$ and $n=1$ terms on the last line of (33)),

$$
\frac{\left[(1-\epsilon)^{2} H H^{\prime}\right]^{\frac{D}{2}-1}}{(4 \pi)^{\frac{D}{2}}} \frac{\Gamma\left(\frac{D+1}{2}+\nu\right) \Gamma\left(\frac{D+1}{2}-\nu\right) \Gamma\left(-\frac{D}{2}\right)}{\Gamma\left(\frac{1}{2}+\nu\right) \Gamma\left(\frac{1}{2}-\nu\right)}\left[\frac{-\frac{D}{2}}{\left(\frac{D-1}{2}\right)^{2}-\nu^{2}}-\frac{y}{4}\right] .
$$

The key to seeing that the infrared divergence of (61) cancels that in (62) is to express both in terms of the small parameter,

$$
\alpha \equiv \nu-\left(\frac{D+1}{2}\right)=\frac{(D \epsilon-2)}{2(1-\epsilon)} .
$$

As before, we extract the common factor of,

$$
\frac{\left[(1-\epsilon)^{2} H H^{\prime}\right]^{\frac{D}{2}-1}}{(4 \pi)^{\frac{D}{2}}} \frac{1}{\Gamma\left(\frac{1}{2}+\nu\right)}=\frac{\left[(1-\epsilon)^{2} H H^{\prime}\right]^{\frac{D}{2}-1}}{(4 \pi)^{\frac{D}{2}}} \frac{1}{\Gamma\left(\frac{D}{2}+1+\alpha\right)} .
$$


The lower limit contribution from (61) is this factor times,

$$
\begin{array}{r}
\frac{2 \Gamma(2 \nu) \Gamma(\nu)}{\Gamma\left(\frac{D-1}{2}\right)}\left[\frac{\left(\eta^{2}+\eta^{\prime 2}\right)}{4(\nu-1) \eta \eta^{\prime}}-\frac{\Delta x^{2}}{2(D-1) \eta \eta^{\prime}}\right] \frac{-z_{0}^{D+1-2 \nu}}{D+1-2 \nu} \\
=\frac{\Gamma(D+1+2 \alpha) \Gamma\left(\frac{D+1}{2}+\alpha\right)}{4 \alpha \Gamma\left(\frac{D+1}{2}\right)}\left[\frac{1}{1+\frac{2 \alpha}{D-1}}\left(\frac{\eta^{2}+\eta^{\prime 2}}{\eta \eta^{\prime}}\right)-\frac{\Delta x^{2}}{\eta \eta^{\prime}}\right]\left(\frac{1}{k_{0}^{2} \eta \eta^{\prime}}\right)^{\alpha}, \\
=\frac{\Gamma(D+1)}{4}\left\{\frac{2-y}{\alpha}+(2-y)\left[2 \psi(D+1)+\psi\left(\frac{D+1}{2}\right)+\ln \left(\frac{1}{k_{0}^{2} \eta \eta^{\prime}}\right)\right]\right. \\
\left.-\frac{2}{D-1}\left(\frac{\eta^{2}+\eta^{\prime 2}}{\eta \eta^{\prime}}\right)+O(\alpha)\right\} .
\end{array}
$$

In contrast, the contribution from (62) is (64) times,

$$
\begin{gathered}
\frac{\Gamma\left(\frac{D+1}{2}+\nu\right) \Gamma\left(\frac{D+1}{2}-\nu\right) \Gamma\left(-\frac{D}{2}\right)}{\Gamma\left(\frac{1}{2}-\nu\right)}\left[\frac{-\frac{D}{2}}{\left(\frac{D-1}{2}\right)^{2}-\nu^{2}}-\frac{y}{4}\right] \\
=-\frac{\Gamma(D+1+\alpha) \Gamma(1-\alpha) \Gamma\left(-\frac{D}{2}\right)}{4 \alpha \Gamma\left(-\frac{D}{2}-\alpha\right)}\left[\frac{2}{\left(1+\frac{\alpha}{D}\right)(1+\alpha)}-y\right], \\
=\frac{\Gamma(D+1)}{4}\left\{-\left(\frac{2-y}{\alpha}\right)+(2-y)\left[-\psi(D+1)+\psi(1)-\psi\left(-\frac{D}{2}\right)\right]\right. \\
\left.+2\left(\frac{D+1}{D}\right)+O(\alpha)\right\} .
\end{gathered}
$$


Adding (66) to (68) and taking $\alpha=0$ (which implies $\epsilon=2 / D$ ) gives,

$$
\begin{array}{r}
\frac{\Gamma(D+1)}{4}\left\{(2-y)\left[\ln \left(\frac{1}{k_{0}^{2} \eta \eta^{\prime}}\right)+\psi(D+1)+\psi\left(\frac{D+1}{2}\right)-\gamma-\psi\left(-\frac{D}{2}\right)\right]\right. \\
\left.-\frac{2}{D-1}\left(\frac{\eta^{2}+\eta^{\prime 2}}{\eta \eta^{\prime}}\right)+2\left(\frac{D+1}{D}\right)\right\}, \\
=\frac{\Gamma(D+1)}{4}\left\{( 2 - y ) \left[\left(\frac{D-2}{D}\right) \ln \left(a a^{\prime}\right)+2 \ln \left[\frac{(D-2) H_{0}}{D k_{0}}\right]-\pi \cot \left(\frac{\pi D}{2}\right)\right.\right. \\
\left.+\psi(D+1)+\psi\left(\frac{D+1}{2}\right)-\gamma-\psi\left(\frac{D}{2}+1\right)\right] \\
\left.-\frac{2}{D-1}\left(\frac{\eta^{2}+\eta^{\prime 2}}{\eta \eta^{\prime}}\right)+2\left(\frac{D+1}{D}\right)\right\} .
\end{array}
$$

We can get the full propagator for $\epsilon=2 / D$ by multiplying (170) by the common factor (64), and then adding the rest of (133) with the now finite $N=0$ correction (51),

$$
\begin{aligned}
& \lim _{\epsilon \rightarrow \frac{2}{D}} i \Delta\left(x ; x^{\prime}\right)= \\
& \quad \frac{\left[\left(1-\frac{2}{D}\right)^{2} H H^{\prime}\right]^{\frac{D}{2}}-1}{(4 \pi)^{\frac{D}{2}}}\left\{\frac{\Gamma\left(\frac{D}{2}+1\right)}{\left(1-\frac{D}{2}\right)\left(-\frac{D}{2}\right)}\left(\frac{4}{y}\right)^{\frac{D}{2}-1}+\frac{\Gamma\left(\frac{D}{2}+2\right)}{\left(2-\frac{D}{2}\right)\left(1-\frac{D}{2}\right)}\left(\frac{4}{y}\right)^{\frac{D}{2}-2}\right. \\
& +\frac{1}{2 !} \frac{\Gamma\left(\frac{D}{2}+3\right)}{\left(3-\frac{D}{2}\right)\left(2-\frac{D}{2}\right)}\left(\frac{4}{y}\right)^{\frac{D}{2}-3}+\frac{\Gamma(D+1)}{4 \Gamma\left(\frac{D}{2}+1\right)}\left[\frac{2(D-1)}{k_{0}^{2} \eta \eta^{\prime}}-\frac{2}{D-1}\left(\frac{\eta}{\eta^{\prime}}+\frac{\eta^{\prime}}{\eta}\right)\right. \\
& \left.+\sum_{n=2}^{\infty}\left[\frac{\left.+2+\frac{2}{D}+(2-y)\left\{\left(1-\frac{2}{D}\right) \ln \left(a a^{\prime}\right)-\pi \cot \left(\frac{\pi D}{2}\right)+K_{D}\right\}\right]}{\left(2-\frac{D}{2}+n\right)\left(1-\frac{D}{2}+n\right)(n+1) !}\left(\frac{y}{4}\right)^{n-\frac{D}{2}+2}-\frac{\Gamma(D+n)}{n(n-1) \Gamma\left(\frac{D}{2}+n\right)}\left(\frac{y}{4}\right)^{n}\right]+O\left(k_{0}^{2}\right)\right\} .
\end{aligned}
$$

Here the constant $K_{D}$ is,

$$
K_{D} \equiv 2 \ln \left[\left(1-\frac{2}{D}\right) \frac{H_{0}}{k_{0}}\right]+\psi(D+1)+\psi\left(\frac{D+1}{2}\right)-\gamma-\psi\left(\frac{D}{2}+1\right) .
$$


As far as we know the literature contains no result against which we can check (71) but its limit in $D=4$ dimensions has been worked out,

$$
\begin{aligned}
\lim _{D \rightarrow 4} \lim _{\epsilon \rightarrow \frac{2}{D}} i \Delta(x ; & \left.x^{\prime}\right)=\frac{H H^{\prime}}{64 \pi^{2}}\left\{\frac{4}{y}+\frac{18}{k_{0}^{2} \eta \eta^{\prime}}-2\left(\frac{\eta}{\eta^{\prime}}+\frac{\eta^{\prime}}{\eta}\right)-11 y+16\right. \\
+ & \left.3(2-y)\left[-\ln (y)+2 \ln \left(\frac{H_{0}}{2 k_{0}}\right)+\frac{1}{2} \ln \left(a a^{\prime}\right)-2 \gamma\right]+O\left(k_{0}^{2}\right)\right\} .
\end{aligned}
$$

This agrees perfectly with equation (3.82) of [24].

We have seen that the lower limit term which corrects the $N=0$ problem in (34) is given by (51). For the $N=1$ problem the corresponding lower limit correction is (61). To see the general pattern, first substitute the relation for $H$ in terms of $\eta$,

$$
H=H_{0}\left[-(1-\epsilon) H_{0} \eta\right]^{\frac{\epsilon}{1-\epsilon}}
$$

This reveals the $N=0$ correction (51) to be constant,

$$
\delta i \Delta_{0} \equiv \frac{\left[(1-\epsilon)^{2} H_{0}^{2}\right]^{\frac{D}{2}-1}}{(4 \pi)^{\frac{D}{2}}} \frac{\Gamma(2 \nu) \Gamma(\nu)}{\Gamma\left(\frac{1}{2}+\nu\right) \Gamma\left(\frac{D-1}{2}\right)} \frac{2(1-\epsilon)}{\epsilon(D-2)}\left[\frac{(1-\epsilon)^{2} H_{0}^{2}}{k_{0}^{2}}\right]^{\frac{\epsilon(D-2)}{2(1-\epsilon)}} .
$$

The same substitution reveals that the $N=1$ correction (61) is quadratic,

$$
\begin{gathered}
\delta i \Delta_{1} \equiv \frac{\left[(1-\epsilon)^{2} H_{0}^{2}\right]^{\frac{D}{2}-1}}{(4 \pi)^{\frac{D}{2}}} \frac{\Gamma(2 \nu) \Gamma(\nu)}{\Gamma\left(\frac{1}{2}+\nu\right) \Gamma\left(\frac{D-1}{2}\right)} \\
\quad \times\left[\frac{k_{0}^{2}\left(\eta^{2}+\eta^{\prime 2}\right)}{4(\nu-1)}-\frac{k_{0}^{2} \Delta x^{2}}{2(D-1)}\right] \frac{2(1-\epsilon)}{(D \epsilon-2)}\left[\frac{(1-\epsilon)^{2} H_{0}^{2}}{k_{0}^{2}}\right]^{\frac{\epsilon(D-2)}{2(1-\epsilon)}} .
\end{gathered}
$$

Both corrections are homogeneous solutions of the propagator equation (4),

$$
\partial_{\mu}\left(\sqrt{-g} g^{\mu \nu} \partial_{\nu} \delta i \Delta_{N}\right)=0
$$

Note that each lower limit correction $\delta i \Delta_{N}$ must separately solve (77) because each goes like a distinct power of $k_{0}$. The freedom to add such homogeneous terms is precisely what is not fixed by just solving the propagator equation rather than using the mode sum. 
We could work out the $\mathrm{N}$-th lower limit correction $\delta i \Delta_{N}$ from the mode sum but that would involve tedious multiplications of corrections from the Bessel function and the two Hankel functions. A simpler technique is to use the fact that the correction must have the form,

$$
\begin{aligned}
\delta i \Delta_{N}=\frac{\left[(1-\epsilon)^{2} H_{0}^{2}\right]^{\frac{D}{2}-1}}{(4 \pi)^{\frac{D}{2}}} \frac{\Gamma(2 \nu) \Gamma(\nu)}{\Gamma\left(\frac{1}{2}+\nu\right) \Gamma\left(\frac{D-1}{2}\right)} \frac{2(1-\epsilon)}{(D-2+2 N) \epsilon-2 N} \\
\times\left[\frac{(1-\epsilon)^{2} H_{0}^{2}}{k_{0}^{2}}\right]^{\frac{\epsilon(D-2)}{2(1-\epsilon)}} k_{0}^{2 N} \sum_{k=0}^{N} \sum_{\ell=0}^{N-k} a_{k \ell} \Delta x^{2 k} \eta^{2 \ell} \eta^{2(N-k-\ell)}
\end{aligned}
$$

Then we determine the coefficients $a_{k \ell}$ by three requirements:

1. The coefficient $a_{N 0}$ derives entirely from the $z^{2 N}$ correction of the Bessel function and, by direct examination of (49), we can see that it is,

$$
a_{N 0}=\frac{(-1)^{N} \Gamma\left(\frac{D-1}{2}\right)}{N ! 4^{N} \Gamma\left(\frac{D-1}{2}+N\right)} ;
$$

2. Symmetry under $\eta \leftrightarrow \eta^{\prime}$ implies,

$$
a_{k \ell}=a_{k(N-k-\ell)}
$$

3. The series must of course solve (77).

The differential equation (77) implies,

$$
\begin{aligned}
0= & {\left[\partial^{2}+\frac{2 \nu-1}{\eta} \partial_{0}\right] \sum_{k=0}^{N} \sum_{\ell=0}^{N-k} a_{k \ell} \Delta x^{2 k} \eta^{2 \ell} \eta^{2(N-k-\ell)} } \\
= & \sum_{k=0}^{N} \sum_{\ell=0}^{N-k} 2 k(2 k+D-3) a_{k \ell} \Delta x^{2 k-2} \eta^{2 \ell} \eta^{\prime 2(N-k-\ell)} \\
& \quad-\sum_{k=0}^{N} \sum_{\ell=0}^{N-k} 4 \ell(\ell-\nu) a_{k \ell} \Delta x^{2 k} \eta^{2 \ell-2} \eta^{2(N-k-\ell)} \\
= & \sum_{k=0}^{N-1} \sum_{\ell=0}^{N-1-k} 2(k+1)(2 k+D-1) a_{k+1 \ell} \Delta x^{2 k} \eta^{2 \ell} \eta^{\prime 2(N-1-k-\ell)} \\
& -\sum_{k=0}^{N-1} \sum_{\ell=0}^{N-1-k} 4(\ell+1)(\ell+1-\nu) a_{k \ell+1} \Delta x^{2 k} \eta^{2 \ell} \eta^{2(N-1-k-\ell)}
\end{aligned}
$$


Hence the coefficients must obey,

$$
(k+1)(2 k+D-1) a_{k+1 \ell}=2(\ell+1)(\ell+1-\nu) a_{k \ell+1} .
$$

The unique solution consistent with the other two of the three properties is,

$$
a_{k \ell}=\left(-\frac{1}{4}\right)^{N} \frac{1}{k ! \ell !(N-k-\ell) !} \frac{\Gamma\left(\frac{D-1}{2}\right) \Gamma^{2}(1-\nu)}{\Gamma\left(k+\frac{D-1}{2}\right) \Gamma(\ell+1-\nu) \Gamma(N-k-\ell+1-\nu)} .
$$

For $N=0$ this gives the known result,

$$
N=0 \quad \Longrightarrow \quad a_{00}=1 \text {. }
$$

A less trivial check is that it also works for $N=1$,

$$
N=1 \quad \Longrightarrow \quad a_{00}=a_{01}=\frac{1}{4(\nu-1)} \quad \text { and } \quad a_{10}=-\frac{1}{2(D-1)} \text {. }
$$

Let us turn now to the decelerating case of $1<\epsilon \leq 2(D-1) / D$ for which the infinite space propagator (33) diverges at the discrete values given in (35). By paralleling what we did for the inflationary case one can show that the lower limit term which corrects the $N=0$ problem is,

$$
\begin{aligned}
\delta i \Delta^{0} & =-\frac{\left[(1-\epsilon)^{2} H H^{\prime}\right]^{\frac{D}{2}-1}}{(4 \pi)^{\frac{D}{2}}} \frac{\Gamma(-2 \nu) \Gamma(-\nu)}{\Gamma\left(\frac{1}{2}-\nu\right) \Gamma\left(\frac{D-1}{2}\right)} 2 \int_{0}^{z_{0}} d z z^{D-2+2 \nu}, \\
& =\frac{\left[(1-\epsilon)^{2} H H^{\prime}\right]^{\frac{D}{2}-1}}{(4 \pi)^{\frac{D}{2}}} \frac{\Gamma(-2 \nu) \Gamma(-\nu)}{\Gamma\left(\frac{1}{2}-\nu\right) \Gamma\left(\frac{D-1}{2}\right)} \frac{2(\epsilon-1)}{2(D-1)-D \epsilon}\left(\frac{1}{k_{0}^{2} \eta \eta^{\prime}}\right)^{\frac{2(D-1)-D \epsilon}{2(\epsilon-1)}}, \\
& =\frac{\left[(1-\epsilon)^{2} H_{0}^{2}\right]^{\frac{D}{2}-1}}{(4 \pi)^{\frac{D}{2}}} \frac{\Gamma(-2 \nu) \Gamma(-\nu)}{\Gamma\left(\frac{1}{2}-\nu\right) \Gamma\left(\frac{D-1}{2}\right)} \frac{2(\epsilon-1)}{2(D-1)-D \epsilon}\left[\frac{(1-\epsilon)^{2} H_{0}^{2}}{k_{0}^{2}}\right]^{\frac{\epsilon(D-2)}{2(1-\epsilon)}}\left(k_{0}^{2} \eta \eta^{\prime}\right)^{2 \nu} .
\end{aligned}
$$

One can easily check that $\left(k_{0}^{2} \eta \eta^{\prime}\right)^{2 \nu}$ solves the homogeneous equation (177). So the full series of these lower limit corrections should take the form,

$$
\begin{aligned}
\delta i \Delta^{N} & =\frac{\left[(1-\epsilon)^{2} H_{0}^{2}\right]^{\frac{D}{2}-1}}{(4 \pi)^{\frac{D}{2}}} \frac{\Gamma(-2 \nu) \Gamma(-\nu)}{\Gamma\left(\frac{1}{2}-\nu\right) \Gamma\left(\frac{D-1}{2}\right)} \frac{2(\epsilon-1)}{2(D-1+N)-(D+2 N) \epsilon} \\
& \times\left[\frac{(1-\epsilon)^{2} H_{0}^{2}}{k_{0}^{2}}\right]^{\frac{\epsilon(D-2)}{2(1-\epsilon)}}\left(k_{0}^{2} \eta \eta^{\prime}\right)^{2 \nu} k_{0}^{2 N} \sum_{k=0}^{N} \sum_{\ell=0}^{N-k} b_{k \ell} \Delta x^{2 k} \eta^{2 \ell} \eta^{2(N-k-\ell)} .
\end{aligned}
$$


We determine the coefficients $b_{k \ell}$ by the same three requirements as the $a_{k \ell}$, although the solution will be different because the ansatz (90) is.

We need to commute the differential operator in (77) through the prefactor of $\left(k_{0}^{2} \eta \eta^{\prime}\right)^{2 \nu}$ in the ansatz (90),

$$
\left[\partial^{2}+\frac{2 \nu-1}{\eta} \partial_{0}\right]\left(k_{0}^{2} \eta \eta^{\prime}\right)^{2 \nu}=\left(k_{0}^{2} \eta \eta^{\prime}\right)^{2 \nu}\left[\partial^{2}+\frac{-2 \nu-1}{\eta} \partial_{0}\right] .
$$

That is a highly significant result because it means the equation the bare series obeys is the same as we already solved for the lower series but with the replacement $\nu \rightarrow-\nu$. So we can write down the answer immediately,

$$
b_{k \ell}=\left(-\frac{1}{4}\right)^{N} \frac{1}{k ! \ell !(N-k-\ell) !} \frac{\Gamma\left(\frac{D-1}{2}\right) \Gamma^{2}(1+\nu)}{\Gamma\left(k+\frac{D-1}{2}\right) \Gamma(\ell+1+\nu) \Gamma(N-k-\ell+1+\nu)} .
$$

It is worth explicitly checking that the lowest $N$ corrections $\delta i \Delta^{N}$ cancel the $\epsilon$ poles in (33) from the upper series (35). From (90) and (92) we see that the $N=0$ correction is,

$$
\delta i \Delta^{0}=\frac{\left[(1-\epsilon)^{2} H H^{\prime}\right]^{\frac{D}{2}-1}}{(4 \pi)^{D / 2}} \frac{\Gamma(-\nu) \Gamma(-2 \nu)}{\Gamma\left(\frac{D-1}{2}\right) \Gamma\left(\frac{1}{2}-\nu\right)} \frac{2(\epsilon-1)}{[2(D-1)-D \epsilon]}\left(\frac{1}{k_{0}^{2} \eta \eta^{\prime}}\right)^{\frac{2(D-1)-D \epsilon}{2(\epsilon-1)}} .
$$

This should cancel the divergence at $\epsilon=2(D-1) / D$ in the $n=0$ term on the last line of (33),

$$
\frac{\left[(1-\epsilon)^{2} H H^{\prime}\right]^{\frac{D}{2}-1}}{(4 \pi)^{D / 2}} \frac{\Gamma\left(1-\frac{D}{2}\right) \Gamma\left(\frac{D-1}{2}+\nu\right) \Gamma\left(\frac{D-1}{2}-\nu\right)}{\Gamma\left(\frac{1}{2}+\nu\right) \Gamma\left(\frac{1}{2}-\nu\right)} .
$$

The relevant small parameter is,

$$
\alpha \equiv \frac{2(D-1)-D \epsilon}{2(\epsilon-1)}=-\frac{D-1}{2}-\nu
$$


Adding (93) to (94) and taking the limit that $\alpha$ vanishes gives,

$$
\begin{gathered}
\lim _{\alpha \rightarrow 0} \frac{\left[(1-\epsilon)^{2} H H^{\prime}\right]^{\frac{D}{2}-1}}{(4 \pi)^{D / 2}} \frac{\Gamma\left(\frac{D-1}{2}+\alpha\right) \Gamma(D-1+2 \alpha)}{\Gamma\left(\frac{D}{2}+\alpha\right) \Gamma\left(\frac{D-1}{2}\right) \alpha}\left\{\left(\frac{1}{k_{0}^{2} \eta \eta^{\prime}}\right)^{\alpha}\right. \\
\left.-\frac{\Gamma\left(\frac{D-1}{2}\right)}{\Gamma\left(\frac{D-1}{2}+\alpha\right)} \frac{\Gamma(D-1+\alpha)}{\Gamma(D-1+2 \alpha)} \frac{\Gamma\left(1-\frac{D}{2}\right)}{\Gamma\left(1-\frac{D}{2}-\alpha\right)} \frac{\Gamma(1-\alpha)}{\Gamma(1)}\right\} . \\
=\frac{\left[\left(1-\frac{2}{D}\right)^{2} H H^{\prime}\right]^{\frac{D}{2}-1}}{(4 \pi)^{D / 2}} \frac{\Gamma(D-1)}{\Gamma\left(\frac{D}{2}\right)}\left\{2 \ln \left[\left(1-\frac{2}{D}\right) \frac{H_{0}}{k_{0}}\right]-\left(1-\frac{2}{D}\right) \ln \left(a a^{\prime}\right)\right. \\
\left.-\pi \cot \left(\frac{D \pi}{2}\right)-\gamma-\psi\left(\frac{D}{2}\right)+\psi(D-1)+\psi\left(\frac{D-1}{2}\right)\right\} .
\end{gathered}
$$

By using $\epsilon-1=1-2 / D$ the final result for the propagator can be expressed in a form that is identical with the de Sitter case (57),

$$
\begin{aligned}
& \lim _{\epsilon \rightarrow \frac{2(D-1)}{D}} i \Delta\left(x ; x^{\prime}\right)= \\
& \frac{\left[(1-\epsilon)^{2} H H^{\prime}\right]^{\frac{D}{2}}-1}{(4 \pi)^{\frac{D}{2}}}\left\{\frac{\Gamma\left(\frac{D}{2}\right)}{\frac{D}{2}-1}\left(\frac{4}{y}\right)^{\frac{D}{2}-1}+\frac{\Gamma\left(\frac{D}{2}+1\right)}{\frac{D}{2}-2}\left(\frac{4}{y}\right)^{\frac{D}{2}-2}+\frac{\Gamma(D-1)}{\Gamma\left(\frac{D}{2}\right)}\left[(1-\epsilon) \ln \left(a a^{\prime}\right)\right.\right. \\
& \left.-\pi \cot \left(\frac{\pi D}{2}\right)+2 \ln \left[|1-\epsilon| \frac{H_{0}}{k_{0}}\right]+\psi\left(\frac{D-1}{2}\right)-\psi\left(\frac{D}{2}\right)+\psi(D-1)-\gamma\right] \\
& \left.+\sum_{n=1}^{\infty}\left[\frac{\Gamma(D-1+n)}{n \Gamma\left(\frac{D}{2}+n\right)}\left(\frac{y}{4}\right)^{n}-\frac{\Gamma\left(\frac{D}{2}+1+n\right)}{\left(2-\frac{D}{2}+n\right)(n+1) !}\left(\frac{y}{4}\right)^{n-\frac{D}{2}+2}\right]+O\left(k_{0}^{2}\right)\right\} .
\end{aligned}
$$

Of course one must remember that in de Sitter $\epsilon=0$ and $H=H_{0}$, so (57) and (98) are only formally the same.

We will content ourselves with working out one more propagator. From (90) and (92) we see that the $N=1$ correction is,

$$
\frac{\left[(1-\epsilon)^{2} H H^{\prime}\right]^{\frac{D}{2}-1}}{(4 \pi)^{D / 2}} \frac{2 \Gamma(-\nu) \Gamma(-2 \nu)}{\Gamma\left(\frac{1}{2}-\nu\right) \Gamma\left(\frac{D-1}{2}\right)}\left(\frac{-1}{4 \eta \eta^{\prime}}\right)\left[\frac{2 \Delta x^{2}}{D-1}+\frac{\eta^{2}+\eta^{\prime 2}}{1+\nu}\right] \frac{\left(k_{0}^{2} \eta \eta^{\prime}\right)^{-\alpha}}{2 \alpha}
$$

where we define the small parameter $\alpha$ as,

$$
\alpha \equiv \frac{2 D-(D+2) \epsilon}{2(\epsilon-1)}=-\frac{D+1}{2}-\nu .
$$


This should cancel the divergences from the $n=0$ and $n=1$ terms on the last line of (33)),

$$
\frac{\left[(1-\epsilon)^{2} H H^{\prime}\right]^{\frac{D}{2}-1}}{(4 \pi)^{D / 2}} \frac{\Gamma\left(\frac{D+1}{2}+\nu\right) \Gamma\left(\frac{D+1}{2}-\nu\right) \Gamma\left(-\frac{D}{2}\right)}{\Gamma\left(\frac{1}{2}+\nu\right) \Gamma\left(\frac{1}{2}-\nu\right)}\left\{-\frac{y}{4}-\frac{\frac{D}{2}}{\left(\frac{D-1}{2}\right)^{2}-\nu^{2}}\right\} .
$$

Adding (99) to (101) and taking $\alpha$ to zero gives,

$$
\begin{aligned}
& \frac{\left[(1-\epsilon)^{2} H H^{\prime}\right]^{\frac{D}{2}-1}}{(4 \pi)^{\frac{D}{2}}} \frac{\Gamma(D+1)}{4 \Gamma\left(\frac{D}{2}+1\right)}\left\{-\frac{2}{D-1}\left(\frac{\eta}{\eta^{\prime}}+\frac{\eta^{\prime}}{\eta}\right)+2+\frac{2}{D}\right. \\
&+(2-y)\left[-\left(\frac{D-2}{D+2}\right) \ln \left(a a^{\prime}\right)+2 \ln \left[\left(\frac{D-2}{D+2}\right) \frac{H_{0}}{k_{0}}\right]-\pi \cot \left(\frac{D \pi}{2}\right)\right. \\
&\left.\left.\quad-\gamma-\psi\left(\frac{D}{2}+1\right)+\psi\left(\frac{D+1}{2}\right)+\psi(D+1)\right]\right\} .
\end{aligned}
$$

By taking advantage of the fact that $(1-\epsilon)=-(D-2) /(D+2)$ we can express the full propagator in a form identical to the $N=1$ result (71) from the lower series,

$$
\begin{aligned}
& \lim _{\epsilon \rightarrow \frac{2 D}{D+2}} i \Delta\left(x ; x^{\prime}\right) \\
& =\frac{\left[(1-\epsilon)^{2} H H^{\prime}\right]^{\frac{D}{2}-1}}{(4 \pi)^{\frac{D}{2}}}\left\{\frac{\Gamma\left(\frac{D}{2}+1\right)}{\left(1-\frac{D}{2}\right)\left(-\frac{D}{2}\right)}\left(\frac{4}{y}\right)^{\frac{D}{2}-1}+\frac{\Gamma\left(\frac{D}{2}+2\right)}{\left(2-\frac{D}{2}\right)\left(1-\frac{D}{2}\right)}\left(\frac{4}{y}\right)^{\frac{D}{2}-2}\right. \\
& +\frac{1}{2 !} \frac{\Gamma\left(\frac{D}{2}+3\right)}{\left(3-\frac{D}{2}\right)\left(2-\frac{D}{2}\right)}\left(\frac{4}{y}\right)^{\frac{D}{2}-3}+\frac{\Gamma(D+1)}{4 \Gamma\left(\frac{D}{2}+1\right)}\left[\frac{2(D-1)}{k_{0}^{2} \eta \eta^{\prime}}-\frac{2}{D-1}\left(\frac{\eta}{\eta^{\prime}}+\frac{\eta^{\prime}}{\eta}\right)\right. \\
& \left.+2+\frac{2}{D}+(2-y)\left\{(1-\epsilon) \ln \left(a a^{\prime}\right)-\pi \cot \left(\frac{\pi D}{2}\right)+C_{D}\right\}\right] \\
& +\sum_{n=2}^{\infty}\left[\frac{\Gamma\left(\frac{D}{2}+2+n\right)}{\left(2-\frac{D}{2}+n\right)\left(1-\frac{D}{2}+n\right)(n+1) !}\left(\frac{y}{4}\right)^{n-\frac{D}{2}+2}\right. \\
& \left.\left.-\frac{\Gamma(D+n)}{n(n-1) \Gamma\left(\frac{D}{2}+n\right)}\left(\frac{y}{4}\right)^{n}\right]+O\left(k_{0}^{2}\right)\right\} \text {. }
\end{aligned}
$$

Here the constant $C_{D}$ is,

$$
C_{D} \equiv 2 \ln \left[|1-\epsilon| \frac{H_{0}}{k_{0}}\right]+\psi(D+1)+\psi\left(\frac{D+1}{2}\right)-\gamma-\psi\left(\frac{D}{2}+1\right) .
$$




\section{The scalar stress-energy tensor}

In this section we shall calculate the expectation value of the scalar stress-energy tensor using the propagator obtained in the previous section. The stress-energy tensor for a scalar field $\varphi$, with the Lagrangian (3), is given by

$$
T_{\mu \nu} \equiv-\frac{2}{\sqrt{-g}} \frac{\delta S}{\delta g^{\mu \nu}}=\partial_{\mu} \varphi \partial_{\nu} \varphi-\frac{1}{2} g_{\mu \nu} g^{\alpha \beta} \partial_{\alpha} \varphi \partial_{\beta} \varphi .
$$

The expectation value with respect to the vacuum state $|\Omega\rangle$ can be written as

$$
\left\langle\Omega\left|T_{\mu \nu}\right| \Omega\right\rangle=\left(\delta_{\mu}^{\rho} \delta_{\nu}^{\sigma}-\frac{1}{2} g_{\mu \nu} g^{\rho \sigma}\right)\left\langle\Omega\left|\partial_{\rho} \varphi \partial_{\sigma} \varphi\right| \Omega\right\rangle=\left.\left(\delta_{\mu}^{\rho} \delta_{\nu}^{\sigma}-\frac{1}{2} g_{\mu \nu} g^{\rho \sigma}\right) \partial_{\rho} \partial_{\sigma}^{\prime} i \Delta\left(x ; x^{\prime}\right)\right|_{x=x^{\prime}},
$$

where $\partial_{\mu}^{\prime} \equiv \frac{\partial}{\partial x^{\prime \mu}}$. The propagator $i \Delta$ is given in terms of the infinite space propagator (30) and the corrections (78) and (90),

$$
i \Delta\left(x ; x^{\prime}\right)=i \Delta_{\infty}\left(x ; x^{\prime}\right)+\sum_{N=0}^{\infty} \delta i \Delta_{N}\left(x ; x^{\prime}\right)+\sum_{N=0}^{\infty} \delta i \Delta^{N}\left(x ; x^{\prime}\right) .
$$

\subsection{The infinite space contribution}

We first consider the contribution to (106) coming from $i \Delta_{\infty}$. From (31) we find that at coincidence $(y \rightarrow 0)$ the following two identities hold

$$
\begin{aligned}
\left.\partial_{\rho} y\right|_{y=0} & =0 \\
\left.\partial_{\rho} \partial_{\sigma}^{\prime} y\right|_{y=0} & =-\frac{2}{\eta^{2}} \eta_{\rho \sigma}=-2(1-\epsilon)^{2} H^{2} g_{\rho \sigma} .
\end{aligned}
$$

Moreover, since in dimensional regularization all $D$ dependent powers of $y$ can be automatically subtracted, we find using (6.131.2) in [8] that the contributions from the hypergeometric function appearing in $i \Delta_{\infty}$ relevant for this calculation are

$$
\begin{aligned}
\left.{ }_{2} F_{1}\left(\frac{D-1}{2}+\nu, \frac{D-1}{2}-\nu ; \frac{D}{2} ; 1-\frac{y}{4}\right)\right|_{y=0} & =\frac{\Gamma\left(1-\frac{D}{2}\right) \Gamma\left(\frac{D}{2}\right)}{\Gamma\left(\frac{1}{2}+\nu\right) \Gamma\left(\frac{1}{2}-\nu\right)} \\
\left.\frac{d}{d y}{ }_{2} F_{1}\left(\frac{D-1}{2}+\nu, \frac{D-1}{2}-\nu ; \frac{D}{2} ; 1-\frac{y}{4}\right)\right|_{y=0} & =-\frac{1}{2 D}\left(\nu^{2}-\left(\frac{D-1}{2}\right)^{2}\right) \frac{\Gamma\left(1-\frac{D}{2}\right) \Gamma\left(\frac{D}{2}\right)}{\Gamma\left(\frac{1}{2}+\nu\right) \Gamma\left(\frac{1}{2}-\nu\right)} .
\end{aligned}
$$


Using these identities we immediately find that

$$
\begin{aligned}
\left.\partial_{\rho} \partial_{\sigma}^{\prime} i \Delta_{\infty}\left(x ; x^{\prime}\right)\right|_{x=x^{\prime}}= & H^{D}|1-\epsilon|^{D} \frac{\Gamma\left(1-\frac{D}{2}\right)}{(4 \pi)^{\frac{D}{2}}} \frac{\Gamma\left(\frac{D-1}{2}+\nu\right) \Gamma\left(\frac{D-1}{2}-\nu\right)}{\Gamma\left(\frac{1}{2}+\nu\right) \Gamma\left(\frac{1}{2}-\nu\right)} \\
& \times\left[\left(\frac{D-1}{2}-\nu\right)^{2} a^{2} \delta_{\rho}^{0} \delta_{\sigma}^{0}+\frac{1}{D}\left(\nu^{2}-\left(\frac{D-1}{2}\right)^{2}\right) g_{\rho \sigma}\right] .
\end{aligned}
$$

Making use of Eq. (106) the one-loop contribution to the stress-energy from $i \Delta_{\infty}$ can be written as,

$$
\begin{aligned}
\left\langle\Omega\left|T_{\mu \nu}\right| \Omega\right\rangle_{\infty}= & \frac{H^{D}|1-\epsilon|^{D}}{(4 \pi)^{D / 2}} \frac{\Gamma\left(1-\frac{D}{2}\right) \Gamma\left(\frac{D-1}{2}+\nu\right) \Gamma\left(\frac{D-1}{2}-\nu\right)}{\Gamma\left(\frac{1}{2}+\nu\right) \Gamma\left(\frac{1}{2}-\nu\right)} \\
& \times\left(\frac{D-1}{2}-\nu\right)\left[\left(\frac{D-1}{2}-\nu\right) a^{2} \delta_{\mu}^{0} \delta_{\nu}^{0}+\frac{1}{D}\left(\frac{(D-1)^{2}}{2}-\nu\right) g_{\mu \nu}\right] .
\end{aligned}
$$

\subsection{The $\epsilon<1$ correction}

Next we consider the $i \delta \Delta_{N}$ contribution (78). We define for convenience

$$
A_{N}=\frac{\left(H_{0}^{2}(1-\epsilon)^{2}\right)^{\frac{D}{2}-1}}{(4 \pi)^{\frac{D}{2}}}\left(\frac{H_{0}^{2}(1-\epsilon)^{2}}{k_{0}^{2}}\right)^{\frac{(D-2) \epsilon}{2(1-\epsilon)}} \frac{\Gamma(2 \nu) \Gamma(\nu)}{\Gamma\left(\frac{1}{2}+\nu\right) \Gamma\left(\frac{D-1}{2}\right)} \frac{-k_{0}^{2 N}}{N+\frac{D-1}{2}-\nu}
$$

such that

$$
\delta i \Delta_{N}\left(x ; x^{\prime}\right)=A_{N} \sum_{k=0}^{N} \sum_{\ell=0}^{N-k} a_{k \ell}(\Delta x)^{2 k} \eta^{2 \ell} \eta^{2(N-k-\ell)} .
$$

Since at coincidence $\Delta x$ is zero, we find that the only nonzero contribution to (106) arises

from $k=1$ when both derivatives hit $\Delta x$, and from $k=0$ when both derivatives hit $\eta$ and $\eta^{\prime}$. Thus at coincidence we have

$$
\begin{aligned}
\left.\partial_{\rho} \partial_{\sigma}^{\prime} \delta i \Delta_{N}\left(x ; x^{\prime}\right)\right|_{x=x^{\prime}}=A_{N} & \left(\sum_{\ell=0}^{N-1} a_{1 \ell}\left(-2 \bar{\eta}_{\rho \sigma} \eta^{2(N-1)}\right)\right. \\
& \left.+\sum_{\ell=0}^{N} a_{0 \ell}\left(4 \ell(N-\ell) \delta_{\rho}^{0} \delta_{\sigma}^{0} \eta^{2(N-1)}\right)\right),
\end{aligned}
$$

where

$$
\bar{\eta}_{\rho \sigma}=\eta_{\rho \sigma}+\delta_{\rho}^{0} \delta_{\sigma}^{0}
$$


Using the expression for $a_{k \ell}$ (85) we find that we can resum the series over $\ell$ to obtain

$$
\begin{aligned}
\left.\partial_{\rho} \partial_{\sigma}^{\prime} \delta i \Delta_{N}\left(x ; x^{\prime}\right)\right|_{x=x^{\prime}}= & A_{N} \frac{(-1)^{N}}{\sqrt{\pi} 4^{\nu}} \frac{\Gamma^{2}(1-\nu) \Gamma\left(N-\frac{1}{2}-\nu\right)}{\Gamma(N) \Gamma(N-\nu) \Gamma(N-2 \nu)} \eta^{2(N-1)} \\
& \times\left(-\frac{1}{D-1} \bar{\eta}_{\rho \sigma}+\frac{N-1}{N-2 \nu} \delta_{\rho}^{0} \delta_{\sigma}^{0}\right),
\end{aligned}
$$

where we made use of the identity,

$$
\sum_{\ell=0}^{N} \frac{N !}{\ell !(N-\ell) !} \frac{\Gamma(N+1-2 \nu)}{\Gamma(\ell+1-\nu) \Gamma(N+1-\ell-\nu)}=\frac{\Gamma(2 N+1-2 \nu)}{\Gamma^{2}(N+1-\nu)} .
$$

From Eqs. (106), (112) and (116) we find the following contribution to the stress-energy tensor from the $N$-th correction,

$$
\begin{aligned}
& \left\langle\Omega\left|T_{\mu \nu}\right| \Omega\right\rangle_{N}=-\frac{H^{D}|1-\epsilon|^{D}}{2(4 \pi)^{D / 2}} \frac{\left(z_{0}^{2}\right)^{N+\frac{D-1}{2}-\nu}}{N+\frac{D-1}{2}-\nu} \frac{\Gamma(1-N+\nu) \Gamma(-N+2 \nu)}{\Gamma\left(\frac{3}{2}-N+\nu\right) \Gamma\left(\frac{D+1}{2}\right) \Gamma(N)} \\
& \times\left[(-(D-1)+2 \nu+(D-2) N) a^{2} \delta_{\mu}^{0} \delta_{\nu}^{0}+\frac{1}{2}(-(D-1)-2(D-3) \nu+2(D-2) N) g_{\mu \nu}\right],
\end{aligned}
$$

where we have transformed the Gamma functions such that they are not singular at the $N$-th pole (47) and we used

$$
\left(H_{0}^{2}(1-\epsilon)^{2}\right)^{\frac{D}{2}-1}\left(\frac{H_{0}^{2}(1-\epsilon)^{2}}{k_{0}^{2}}\right)^{\frac{(D-2) \epsilon}{2(1-\epsilon)}} \frac{1}{\eta^{2}}=H^{D}|1-\epsilon|^{D}\left(z_{0}^{2}\right)^{\frac{D-1}{2}-\nu} a^{2},
$$

with $z_{0}=k_{0}|\eta|$ at coincidence.

\subsection{The $\epsilon>1$ correction}

Finally we consider the correction due to $\delta i \Delta^{N}$ given by Eq. (90). We define for convenience

$$
B_{N}=\frac{\left(H_{0}^{2}(1-\epsilon)^{2}\right)^{\frac{D}{2}-1}}{(4 \pi)^{\frac{D}{2}}}\left(\frac{H_{0}^{2}(1-\epsilon)^{2}}{k_{0}^{2}}\right)^{\frac{(D-2) \epsilon}{2(1-\epsilon)}} \frac{\Gamma(-2 \nu) \Gamma(-\nu)}{\Gamma\left(\frac{1}{2}-\nu\right) \Gamma\left(\frac{D-1}{2}\right)} \frac{-k_{0}^{2 N}}{N+\frac{D-1}{2}+\nu},
$$

such that

$$
\delta i \Delta^{N}\left(x ; x^{\prime}\right)=B_{N}\left(k_{0}^{2} \eta \eta^{\prime}\right)^{2 \nu} \sum_{k=0}^{N} \sum_{\ell=0}^{N-k} b_{k \ell}(\Delta x)^{2 k} \eta^{2 \ell} \eta^{2(N-k-\ell)} .
$$


The calculation is similar as in the $\epsilon<1$ case, and it yields:

$$
\begin{aligned}
\left.\partial_{\rho} \partial_{\sigma}^{\prime} \delta i \Delta^{N}\left(x ; x^{\prime}\right)\right|_{x=x^{\prime}}=B_{N}\left(k_{0}^{2} \eta^{2}\right)^{2 \nu} & \left(\sum_{\ell=0}^{N-1} b_{1 \ell}\left(-2 \bar{\eta}_{\rho \sigma} \eta^{2(N-1)}\right)\right. \\
& \left.+\sum_{\ell=0}^{N} b_{0 \ell}\left(4(\ell+\nu)(N-\ell+\nu) \delta_{\rho}^{0} \delta_{\sigma}^{0} \eta^{2(N-1)}\right)\right) .
\end{aligned}
$$

Summing over $\ell$ gives

$$
\begin{aligned}
\left.\partial_{\rho} \partial_{\sigma}^{\prime} \delta i \Delta^{N}\left(x ; x^{\prime}\right)\right|_{x=x^{\prime}}= & B_{N}\left(k_{0}^{2} \eta^{2}\right)^{2 \nu} \frac{(-1)^{N} 4^{\nu}}{\sqrt{\pi}} \frac{\Gamma^{2}(1+\nu) \Gamma\left(N-\frac{1}{2}+\nu\right)}{\Gamma(N) \Gamma(N+\nu) \Gamma(N+2 \nu)} \eta^{2(N-1)} \\
& \times\left(-\frac{1}{D-1} \bar{\eta}_{\rho \sigma}+\frac{N-1+2 \nu}{N} \delta_{\rho}^{0} \delta_{\sigma}^{0}\right)
\end{aligned}
$$

resulting in the following contribution to the stress-energy tensor,

$$
\begin{aligned}
\left\langle\Omega\left|T_{\mu \nu}\right| \Omega\right\rangle^{N} & =-\frac{H^{D}|1-\epsilon|^{D}}{2(4 \pi)^{D / 2}} \frac{\left(z_{0}^{2}\right)^{N+\frac{D-1}{2}+\nu}}{N+\frac{D-1}{2}+\nu} \frac{\Gamma(1-N-\nu) \Gamma(1-N-2 \nu)}{\Gamma\left(\frac{3}{2}-N-\nu\right) \Gamma\left(\frac{D+1}{2}\right) \Gamma(N+1)} \\
& \times\left[((D-1)(1-2 \nu)-(D-2) N) a^{2} \delta_{\mu}^{0} \delta_{\nu}^{0}+\frac{1}{2}((D-1)(1-2 \nu)-2(D-2) N) g_{\mu \nu}\right] .
\end{aligned}
$$

\subsection{Renormalization}

The total one-loop stress energy tensor is the sum of the three contributions (111), (118) and (124),

$$
\left\langle\Omega\left|T_{\mu \nu}\right| \Omega\right\rangle=\left\langle\Omega\left|T_{\mu \nu}\right| \Omega\right\rangle_{\infty}+\sum_{N=0}^{\infty}\left\langle\Omega\left|T_{\mu \nu}\right| \Omega\right\rangle_{N}+\sum_{N=0}^{\infty}\left\langle\Omega\left|T_{\mu \nu}\right| \Omega\right\rangle^{N} .
$$

Note that the ultraviolet divergence (which in dimensional regularization appears as a term

multiplying $1 /(D-4)$ ) is confined to (111). Indeed, when expanded around $D=4$, Eq. (111) gives,

$$
\begin{aligned}
\left\langle\Omega\left|T_{\mu \nu}\right| \Omega\right\rangle_{\infty}= & \left\{-\frac{(2-\epsilon) \epsilon H^{D}}{8 \pi^{2}} \frac{1}{D-4}\right. \\
& \left.-\frac{(2-\epsilon) \epsilon H^{4}}{16 \pi^{2}}\left[4-\epsilon+\gamma+\ln \left(\frac{(1-\epsilon)^{2}}{4 \pi}\right)+\psi\left(\frac{1}{1-\epsilon}\right)+\psi\left(-\frac{\epsilon}{1-\epsilon}\right)\right]\right\} \\
& \times\left[\epsilon a^{2} \delta_{\mu}^{0} \delta_{\nu}^{0}+\left(\epsilon-\frac{3}{4}\right) g_{\mu \nu}\right]+\frac{(2-\epsilon) \epsilon H^{4}}{128 \pi^{2}} g_{\mu \nu}+\mathcal{O}(D-4) .
\end{aligned}
$$


It is known that this theory can be renormalized by the $R^{2}$ counterterm only. Indeed, taking a functional derivative with respect to $g^{\mu \nu}$ of the counterterm action results in

$$
-\frac{2}{\sqrt{-g}} \frac{\delta}{\delta g^{\mu \nu}} \int d^{D} x \sqrt{-g} \alpha R^{2}=\alpha\left(4 \nabla_{\mu} \nabla_{\nu} R-4 g_{\mu \nu} \square R+g_{\mu \nu} R^{2}-4 R R_{\mu \nu}\right) .
$$

Making use of the corresponding expressions for $R$ and $R_{\mu \nu}$ in FLRW spaces (see e.g. Ref. [3]) this evaluates to

$$
\begin{aligned}
-\frac{2}{\sqrt{-g}} \frac{\delta}{\delta g^{\mu \nu}} \int & d^{D} x \sqrt{-g} R^{2}=144(2-\epsilon) \epsilon H^{4}\left[\epsilon a^{2} \delta_{\mu}^{0} \delta_{\nu}^{0}+\left(\epsilon-\frac{3}{4}\right) g_{\mu \nu}\right] \\
& -H^{4}\left(48 \epsilon\left(1-4 \epsilon+\epsilon^{2}\right) a^{2} \delta_{\mu}^{0} \delta_{\nu}^{0}-12\left(3-22 \epsilon+22 \epsilon^{2}-4 \epsilon^{3}\right) g_{\mu \nu}\right)(D-4) \\
& +\mathcal{O}\left((D-4)^{2}\right) .
\end{aligned}
$$

Note that the $H^{D}$ term in Eq. (126) can be expanded as,

$$
H^{D}=H^{4} \mu^{D-4}\left[1+\frac{D-4}{2} \ln \left(\frac{H^{2}}{\mu^{2}}\right)\right]+\mathcal{O}\left((D-4)^{2}\right),
$$

where $\mu$ is an arbitrary renormalization scale. From Eqs. (126) and (128) we see that the divergence in (126) is canceled by

$$
\alpha=\frac{\mu^{D-4}}{1152 \pi^{2}(D-4)}
$$

where $\mu$ controls the undetermined finite part of $\alpha$. The renormalized stress-energy tensor can be now easily obtained

$$
\begin{aligned}
\left\langle\Omega\left|T_{\mu \nu}\right| \Omega\right\rangle & =-\frac{H^{4}}{48 \pi^{2}}\left\{\left(2+16 \epsilon-16 \epsilon^{2}+3 \epsilon^{3}\right) a^{2} \delta_{\mu}^{0} \delta_{\nu}^{0}-\frac{1}{8}\left(12+62 \epsilon-215 \epsilon^{2}+146 \epsilon^{3}-24 \epsilon^{4}\right) g_{\mu \nu}\right. \\
+ & 3 \epsilon(2-\epsilon)\left(\gamma+\psi\left(-\frac{\epsilon}{1-\epsilon}\right)+\psi\left(\frac{1}{1-\epsilon}\right)+\ln \left(\frac{(1-\epsilon)^{2} H^{2}}{4 \pi \mu^{2}}\right)\right) \\
& \left.\times\left[\epsilon a^{2} \delta_{\mu}^{0} \delta_{\nu}^{0}+\left(\epsilon-\frac{3}{4}\right) g_{\mu \nu}\right]\right\} \\
+ & \sum_{N=0}^{\infty}\left(\left\langle\Omega\left|T_{\mu \nu}\right| \Omega\right\rangle_{N}+\left\langle\Omega\left|T_{\mu \nu}\right| \Omega\right\rangle^{N}\right)_{D \rightarrow 4}
\end{aligned}
$$


where the terms in the last line denote the $D \rightarrow 4$ limit of Eqs. (118) and (124).

The sums over $N$ can be performed and the final result can than be recast in terms of a hypergeometric function ${ }_{2} F_{3}$. This is a useful procedure for studying the ultraviolet behavior of the corrections (when the cutoff $\left|z_{0}\right| \rightarrow \infty$ ). Since here we are primarily interested in the infrared sector $\left|z_{0}\right| \ll 1$, we shall not perform the summation over $N$.

\subsection{Resolving the divergencies of the digamma functions}

Even though the ultraviolet divergences have been removed by dimensional renormalization, the renormalized stress-energy (131) still seems to diverge at the poles of the (di)gamma functions (34 35) (see also Eqs. (45 47)). We shall now show that these divergences are only apparent however, and that they are canceled by the correction terms in (131) given by (118) and (124), precisely as there were designed to do. 1

Let us first consider the case $\epsilon<1$. Expanding (34) around the $N$-th pole we have

$$
-\frac{\epsilon}{1-\epsilon}=-N+\delta \Rightarrow \epsilon=\frac{N-\delta}{N+1-\delta}, \quad \nu=\frac{3}{2}+N-\delta,
$$

where $N$ is a positive integer and $\delta$ is an infinitesimal quantity. In this case the first digamma function in (131) diverges, and its contribution to the stress-energy tensor is

$$
\frac{H^{4}}{16 \pi^{2}} \frac{N(N+2)}{(N+1)^{2}} \frac{1}{\delta}\left[\epsilon a^{2} \delta_{\mu}^{0} \delta_{\nu}^{0}+\left(\epsilon-\frac{3}{4}\right) g_{\mu \nu}\right]+\mathcal{O}\left(\delta^{0}\right)
$$

where we used

$$
\psi(-N+\delta)=-\frac{1}{\delta}+\mathcal{O}\left(\delta^{0}\right)
$$

To check that our construction works next we rewrite the $N$-th term (118) by using (132)

$$
\begin{aligned}
\left\langle\Omega\left|T_{\mu \nu}\right| \Omega\right\rangle_{N} \stackrel{D \rightarrow 4}{\longrightarrow} & -\frac{H^{4}}{32 \pi^{2}(1+N-\delta)^{4}} \frac{\left(z_{0}^{2}\right)^{\delta}}{\delta} \frac{\Gamma\left(\frac{5}{2}-\delta\right) \Gamma(N+3-2 \delta)}{\Gamma(3-\delta) \Gamma\left(\frac{5}{2}\right) \Gamma(N)} \\
& \times\left[2(2 N-\delta) a^{2} \delta_{\mu}^{0} \delta_{\nu}^{0}+(N-3+\delta) g_{\mu \nu}\right] .
\end{aligned}
$$

When expanded in powers of $\delta$ this gives,

$$
\begin{aligned}
\left\langle\Omega\left|T_{\mu \nu}\right| \Omega\right\rangle_{N} \stackrel{D \rightarrow 4}{\longrightarrow} & -\frac{H^{4}}{16 \pi^{2}} \frac{N(N+2)}{(N+1)^{2}}\left[\frac{1}{\delta}+\ln \left(z_{0}^{2}\right)-\psi\left(\frac{5}{2}\right)-2 \psi(N+3)+\psi(3)+\frac{4}{N+1}\right] \\
& \times\left[\frac{N}{N+1} a^{2} \delta_{\mu}^{0} \delta_{\nu}^{0}+\frac{N-3}{4(N+1)} g_{\mu \nu}-\frac{\delta}{2(N+1)}\left(a^{2} \delta_{\mu}^{0} \delta_{\nu}^{0}-\frac{1}{2} g_{\mu \nu}\right)\right]+\mathcal{O}(\delta) .(130
\end{aligned}
$$

\footnotetext{
${ }^{1}$ One might think that the second digamma function in (131) has a simple pole also at $\epsilon=2$, but that is canceled by the $(2-\epsilon)$ prefactor.
} 
Note that close to the pole $N /(N+1)=\epsilon+\mathcal{O}(\delta)$ and $(N-3) /[4(N+1)]=\epsilon-(3 / 4)+\mathcal{O}(\delta)$, such that the tensor structures in (136) and (133) are (to leading order in $\delta$ ) identical. Moreover, by comparing Eq. (136) with (133) we see that the $\mathcal{O}(1 / \delta)$ terms cancel, as required. The resulting leading order contribution to the one-loop stress-energy tensor is finite and depends logarithmically on the scale factor as $\propto H^{4} a^{2}[\ln (a)+$ const.], where the constant term contains a logarithm of the cutoff $k_{0}$.

In the case when $\epsilon>1$ the singularities of the digamma functions in the stress-energy tensor (131) conform with the poles (35). As above, expanding around the poles

$$
\frac{1}{1-\epsilon}=-2-N+\delta \Rightarrow \nu=-\frac{3}{2}-N+\delta, \quad \epsilon=\frac{N+3-\delta}{N+2-\delta},
$$

we obtain the following contribution to (131) from the digamma function:

$$
\frac{H^{4}}{16 \pi^{2}} \frac{(N+1)(N+3)}{(N+2)^{2}} \frac{1}{\delta}\left(\epsilon a^{2} \delta_{\mu}^{0} \delta_{\nu}^{0}+\left(\epsilon-\frac{3}{4}\right) g_{\mu \nu}\right)+\mathcal{O}\left(\delta^{0}\right)
$$

On the other hand, the correction (124) contributes the following to the scalar stressenergy (131)

$$
\begin{aligned}
\left\langle\Omega\left|T_{\mu \nu}\right| \Omega\right\rangle^{N} \stackrel{D \rightarrow 4}{\longrightarrow} & -\frac{H^{4}}{16 \pi^{2}} \frac{(N+1)(N+3)}{(N+2)^{2}}\left[\frac{1}{\delta}+\ln \left(z_{0}^{2}\right)-\psi\left(\frac{5}{2}\right)-2 \psi(N+4)+\psi(3)+\frac{4}{N+2}\right] \\
& \times\left[\frac{N+3}{N+2} a^{2} \delta_{\mu}^{0} \delta_{\nu}^{0}+\frac{N+6}{4(N+2)} g_{\mu \nu}-\frac{3 \delta}{2(N+2)}\left(a^{2} \delta_{\mu}^{0} \delta_{\nu}^{0}+\frac{1}{2} g_{\mu \nu}\right)\right]+\mathcal{O}(\delta) .
\end{aligned}
$$

Noting that here $(N+3) /(N+2)=\epsilon+\mathcal{O}(\delta)$ and $(N+6) /[4(N+2)]=\epsilon-(3 / 4)+\mathcal{O}(\delta)$, we see that the pole contributions $\mathcal{O}(1 / \delta)$ in (139) and (138) cancel, resulting again in a finite contribution to the scalar one-loop stress-energy of the form, $\propto H^{4} a^{2}[\ln (a)+$ const.].

\section{Discussion}

We studied the propagator of the massless, minimally coupled scalar on a spatially flat, FLRW background of arbitrary dimension and constant $\epsilon=-\dot{H} / H^{2}$. A previous result (33), first derived [3, 4] by solving the propagator equation (4), was seen to agree with the Bunch-Davies mode sum for infinite space. It diverges at discrete values of $\epsilon$ (34 35), even away from coincidence and with the dimensional regularization in effect. The origin of these divergences derives from the way dimensional regularization treats the infrared divergences that the mode sum possesses for all $\epsilon$ in the range $0 \leq \epsilon \leq 2(D-1) / D$. For most values 
of $\epsilon$ in this range the infrared divergences are of the power law type that are set to zero by the automatic subtraction of dimensional regularization. The infrared divergences become logarithmic for the special values of $\epsilon$ given by the two series (34) and (35), which is why the infinite space propagator (33) diverges for these values.

Of course one should never subtract infrared divergences, even power law ones. Infrared divergences signify an unphysical feature of whatever question is being posed and the correct way to avoid them is by making appropriate changes in the question. The unphysical feature of the infinite space propagator is that no local observer could prepare the initial state in coherent Bunch-Davies vacuum over more than a Hubble volume. A more realistic situation is attained either by preparing the initially super-horizon modes in some less singular state or else by simply not having any initially super-horizon modes.

We implemented the latter fix, making the integral approximation to the discrete mode sum but with a nonzero lower limit. The resulting propagator (49) could be expressed as the old result (33), minus a series of homogeneous solutions. Canceling the infrared divergences requires only the most singular contributions. For the inflationary case of $0 \leq \epsilon<1$ our results for the corrections are (178) and (85); for the decelerating case of $1<\epsilon \leq 2(D-1) / D$ they are (190) and (92). We showed explicitly that the first two divergences of (34) and the first two divergences of (35) are canceled in this way, and we obtained the resulting propagators with dimensional regularization still in effect. We also showed that these results agree with special cases which have been reported in the literature [21, 24]. Moreover as an example we calculated the one-loop expectation value of the stress-energy tensor and showed that the divergences as discussed in [4] are canceled and replaced with a term that grows as the logarithm of the infrared cutoff.

The case of $\epsilon=1$ deserves special comment. From the convergence of the lower singularities (34) and the upper ones (35) it might seem that the infinite space mode sum is highly infrared divergent. However, this is just an illusion derived from the fact that our convention for the zero of conformal time shifts discontinuously at $\epsilon=1$. If one takes the limit $\epsilon \rightarrow 1$ while holding fixed the co-moving time and the initial Hubble parameter then the mode functions have a perfectly well-behaved form,

$$
\lim _{\epsilon \rightarrow 1} u(t, k)=\frac{a^{1-\frac{D}{2}}}{\sqrt{2 H_{0}}}\left[\frac{k^{2}}{H_{0}^{2}}-\left(\frac{D-2}{2}\right)^{2}\right]^{-\frac{1}{4}} \exp \left[-i \sqrt{\frac{k^{2}}{H_{0}^{2}}-\left(\frac{D-2}{2}\right)^{2}} \ln (a)\right] .
$$

The propagator also has a smooth limit,

$$
\lim _{\epsilon \rightarrow 1} i \Delta_{\infty}\left(x ; x^{\prime}\right)=\frac{\left[H H^{\prime}\right]^{\frac{D}{2}-1}}{(4 \pi)^{\frac{D}{2}}} \Gamma\left(\frac{D}{2}-1\right)\left(\frac{4}{Y}\right)^{\frac{D}{2}-1}
$$


where we define the symbol $Y$ as

$$
Y\left(x ; x^{\prime}\right) \equiv H_{0}^{2} \Delta x^{2}-\left(\left|\ln \left(\frac{a}{a^{\prime}}\right)\right|-i \varepsilon\right)^{2} .
$$

An important distinction exists between the inflationary case of $0 \leq \epsilon<1$ and the case of deceleration. The reason for this is that the parameter used to characterize the infrared cutoff, $z_{0}$, approaches zero in an inflationary space, but it grows without bound in a decelerating space-time. In an inflationary space-time the physical wavelength associated with the infrared cutoff, $a(t) / k_{0}$, grows faster than the Hubble radius. Thus a super-Hubble cutoff stays super-Hubble at all times. In contrast, for a decelerating universe the Hubble radius grows faster than $a(t) / k_{0}$ and therefore an initial super-Hubble cutoff will eventually enter the Hubble radius. Hence the effect of the cutoff becomes more and more profound as time evolves. This behavior can be seen nicely from the expectation value of the stressenergy tensor (131). The corrections from $\delta i \Delta_{N}$ and $\delta i \Delta^{N}$ have the following respective time dependences:

$$
\begin{aligned}
H^{4}\left(z_{0}^{2}\right)^{N-\frac{\epsilon}{1-\epsilon}} & \propto a^{-2[\epsilon+N(1-\epsilon)]} \\
H^{4}\left(z_{0}^{2}\right)^{N+\frac{3-2 \epsilon}{1-\epsilon}} & \propto a^{-2[3+N(1-\epsilon)]} .
\end{aligned}
$$

This behavior should be compared to the tree level contribution to the equation of motion, which follows from the Friedmann equations, and scales as $H^{2} \propto a^{-2 \epsilon}$ and $\dot{H} \propto a^{-2 \epsilon}$. Thus we see that for an inflationary space, $\epsilon<1$, all corrections $N \geq 1$ decay faster with time than the tree-level contributions, while the $N=0$ correction scales with time equally as $H^{2}$ and $\dot{H}$. For a decelerating space $\epsilon>1$ however, there are infinitely many values of $N$ for which both corrections grow indefinitely as the universe expands. 2

Thus we conclude that for an inflationary universe, the initial conditions become less visible as time progresses. In this case it does not matter much which of the two fixes for the infrared problems of Section 3 is employed, or precisely how it is implemented, while the opposite is true for a decelerating universe. Although we have worked out the fix based on a finite spatial manifold, the absence of evidence for a finite size to the universe suggests that the more physically relevant fix for deceleration is the one based on a less singular ensemble of initially super-horizon modes [15]. Because the initial condition becomes progressively more visible for deceleration, the physically relevant choice is probably the one consistent with at least 60 e-foldings of primordial inflation. Note that modes which experience first

\footnotetext{
${ }^{2}$ In order to get the correct late time behavior in this case, one would have to sum the series in $N$ and consider the asymptotic behavior of the resulting hypergeometric function ${ }_{2} F_{3}$ in the limit when $\left|z_{0}\right| \gg 1$. Since that limit corresponds to a sub-Hubble cutoff, it is not of great interest to us.
} 
horizon crossing will be even more infrared singular than for Bunch-Davies vacuum. It would only be for currently unobservable super-horizon modes that one could use the less singular mode functions needed to regulate the infrared divergence. Hence one expects the infrared behavior of the physically relevant propagator for deceleration to be strongly influenced by the initial condition provided by primordial inflation.

\section{Acknowledgements}

We are grateful for conversations on this subject with N. C. Tsamis. This work was partially supported by FOM grant 07PR2522, by Utrecht University, by NSF grant PHY0653085, and by the Institute for Fundamental Theory at the University of Florida.

\section{References}

[1] L. P. Grishchuk, Sov. Phys. JETP 40 (1975) 409 [Zh. Eksp. Teor. Fiz. 67 (1974) 825].

[2] T. Janssen and T. Prokopec, Class. Quant. Grav. 25 (2008) 055007 arXiv:0707.3919 $[\mathrm{gr}-\mathrm{qc}]$.

[3] T. Janssen, S. P. Miao and T. Prokopec, arXiv:0807.0439 [gr-qc].

[4] T. Janssen and T. Prokopec, arXiv:0807.0447 [gr-qc].

[5] N. C. Tsamis and R. P. Woodard, Class. Quant. Grav. 18 (2001) 83 [arXiv:hep$\mathrm{ph} / 0007167]$.

[6] N. C. Tsamis and R. P. Woodard, Class. Quant. Grav. 20 (2003) 5205 [arXiv:astro$\mathrm{ph} / 0206010]$.

[7] N. D. Birrell and P. C. W. Davies, "Quantum Fields In Curved Space," Cambridge, Uk: Univ. Pr. (1982)

[8] I. S. Gradshteyn and I. M. Ryzhik, "Table of Integrals, Series and Products, 4th Edition" New York, USA: Academic Press (1965)

[9] N. A. Chernikov and E. A. Tagirov, Annales Poincare Phys. Theor. A 9 (1968) 109.

[10] T. S. Bunch and P. C. W. Davies, Proc. Roy. Soc. Lond. A 357 (1977) 381.

[11] L. H. Ford and L. Parker, Phys. Rev. D 16 (1977) 245. 
[12] G. 't Hooft and M. J. G. Veltman, Nucl. Phys. B 44 (1972) 189.

[13] C. G. Bollini and J. J. Giambiagi, Nuovo Cim. B 12 (1972) 20.

[14] S. Weinberg, "The Quantum theory of fields. Vol. 1: Foundations," Cambridge, UK: Univ. Pr. (1995)

[15] A. Vilenkin, Nucl. Phys. B 226, 527 (1983).

[16] S. A. Fulling, M. Sweeny and R. M. Wald, Commun. Math. Phys. 63 (1978) 257.

[17] N. C. Tsamis and R. P. Woodard, Class. Quant. Grav. 11, 2969 (1994).

[18] N. C. Tsamis and R. P. Woodard, Phys. Rev. D 54, 2621 (1996) [arXiv:hep-ph/9602317].

[19] S. P. Miao and R. P. Woodard, Class. Quant. Grav. 23, 1721 (2006) [arXiv:grqc/0511140];

S. P. Miao, arXiv:0705.0767 [hep-th].

[20] E. O. Kahya and R. P. Woodard, Phys. Rev. D 76, 124005 (2007) [arXiv:0709.0536 $[\mathrm{gr}-\mathrm{qc}]]$.

[21] V. K. Onemli and R. P. Woodard, Class. Quant. Grav. 19, 4607 (2002) [arXiv:grqc/0204065];

V. K. Onemli and R. P. Woodard, Phys. Rev. D 70, 107301 (2004) [arXiv:grqc/0406098].

[22] T. Brunier, V. K. Onemli and R. P. Woodard, Class. Quant. Grav. 22, 59 (2005) [arXiv:gr-qc/0408080].

[23] T. Prokopec, N. C. Tsamis and R. P. Woodard, Class. Quant. Grav. 24, 201 (2007) [arXiv:gr-qc/0607094];

T. Prokopec, N. C. Tsamis and R. P. Woodard, Phys. Rev. D 78, 043523 (2008) arXiv:0802.3673 [gr-qc].

[24] J. Iliopoulos, T. N. Tomaras, N. C. Tsamis and R. P. Woodard, Nucl. Phys. B 534, 419 (1998) [arXiv:gr-qc/9801028].

[25] T. Prokopec, O. Tornkvist and R. P. Woodard, Phys. Rev. Lett. 89, 101301 (2002) [arXiv:astro-ph/0205331];

T. Prokopec, O. Tornkvist and R. P. Woodard, "One loop vacuum polarization in a locally de Sitter background," Annals Phys. 303, 251 (2003) [arXiv:gr-qc/0205130]; 
T. Prokopec and R. P. Woodard, JHEP 0310, 059 (2003) [arXiv:astro-ph/0309593];

N. C. Tsamis and R. P. Woodard, Annals Phys. 321, 875 (2006) [arXiv:gr-qc/0506056];

E. O. Kahya and R. P. Woodard, Phys. Rev. D 72, 104001 (2005) [arXiv:gr-qc/0508015];

S. P. Miao and R. P. Woodard, Class. Quant. Grav. 25, 145009 (2008) [arXiv:0803.2377 [gr-qc]]. 